\title{
Holographic c-theorems in arbitrary dimensions
}

\author{
Robert C. Myers ${ }^{1}$ and Aninda Sinha ${ }^{1,2}$ \\ ${ }^{1}$ Perimeter Institute for Theoretical Physics \\ Waterloo, Ontario N2L 2Y5, Canada \\ ${ }^{2}$ Centre for High Energy Physics, Indian Institute of Science, \\ C.V.Raman Avenue, Bangalore 560012, India
}

\begin{abstract}
We re-examine holographic versions of the c-theorem and entanglement entropy in the context of higher curvature gravity and the AdS/CFT correspondence. We select the gravity theories by tuning the gravitational couplings to eliminate nonunitary operators in the boundary theory and demonstrate that all of these theories obey a holographic c-theorem. In cases where the dual CFT is even-dimensional, we show that the quantity that flows is the central charge associated with the A-type trace anomaly. Here, unlike in conventional holographic constructions with Einstein gravity, we are able to distinguish this quantity from other central charges or the leading coefficient in the entropy density of a thermal bath. In general, we are also able to identify this quantity with the coefficient of a universal contribution to the entanglement entropy in a particular construction. Our results suggest that these coefficients appearing in entanglement entropy play the role of central charges in odddimensional CFT's. We conjecture a new c-theorem on the space of odd-dimensional field theories, which extends Cardy's proposal for even dimensions. Beyond holography, we were able to show that for any even-dimensional CFT, the universal coefficient appearing the entanglement entropy which we calculate is precisely the A-type central charge.
\end{abstract}




\section{Contents}

1. Introduction 1

2. Holographic c-theorems - Take One 5

3. Holographic c-theorems - Take Two 10

4. $a_{d}^{*}$ and Entanglement Entropy - Take One 19

5. $a_{d}^{*}$ and Entanglement Entropy - Take Two 23

5.1 Conical defect in $\mathrm{AdS}_{d+1}$

5.2 AdS equations of motion 30

5.3 A-type trace anomaly 32

5.4 Entanglement entropy without holography 33

5.5 Counting degrees of freedom 36

6. Other central charges 38

7. Discussion 42

A. RG flows $\quad 49$

B. More general couplings $\quad 50$

C. Non-relativistic $c$-theorems $\quad 53$

\section{Introduction}

Zamolodchikov's c-theorem [1] states that for quantum field theories (QFT's) in two dimensions, there exists a positive definite real function $c(g)$ on the space of couplings $g^{i}$, which satisfies the following three properties: $i) c(g)$ is monotonically decreasing along renormalization group ( $\mathrm{RG}$ ) flows. $i i) c(g)$ is stationary at $\mathrm{RG}$ fixed points $g^{i}=\left(g^{*}\right)^{i}$, i.e., $\partial c(g) /\left.\partial g^{i}\right|_{g^{*}}=0$. iii) At RG fixed points, $c(g)$ equals the central charge of the corresponding conformal field theory $(\mathrm{CFT})$. This remarkable result requires only very 
simple conditions of the QFT's with the proof relying only on the Euclidean group of symmetries, the existence of a conserved stress-energy tensor and unitarity in the field theory. A direct consequence of the c-theorem is that in any renormalization group (RG) flow connecting two fixed points,

$$
(c)_{\mathrm{UV}} \geq(c)_{\mathrm{IR}}
$$

That is, the central charge of the CFT describing the ultraviolet fixed point is larger than (or equal to) that at the infrared fixed point. An intuitive understanding of this result comes from the interpretation that the central charge provides a measure of the number of degrees of freedom of the underlying CFT. Its decrease along the RG flow can then be seen as a consequence of integrating out high-energy degrees of freedom in the Wilsonian approach to the renormalization group.

There have been various suggestions on how such a result might extend to quantum field theories in higher $d$. One approach refers to the trace anomaly which is fixed by the central charge $c$ for two-dimensional CFT's [2], i.e.,

$$
\left\langle T_{a}^{a}\right\rangle=-\frac{c}{12} R
$$

Turning to $d=4$, this expression generalizes to [2]

$$
\left\langle T_{a}^{a}\right\rangle=\frac{c}{16 \pi^{2}} W_{a b c d} W^{a b c d}-\frac{a}{16 \pi^{2}}\left(R_{a b c d} R^{a b c d}-4 R_{a b} R^{a b}+R^{2}\right)-\frac{a^{\prime}}{16 \pi^{2}} \nabla^{2} R
$$

where $W_{a b c d} W^{a b c d}=R_{a b c d} R^{a b c d}-2 R_{a b} R^{a b}+R^{2} / 3$ is the square of the four-dimensional Weyl tensor and the expression in the second term is proportional to the four-dimensional Euler density. Hence, the question naturally arises: do any of $c, a$ or $a^{\prime}$ satisfy a ctheorem under RG flows? Since $a^{\prime}$ is scheme dependent [3] and cannot be defined globally [4], it is not a useful charge to consider. With regards to the four-dimensional central charge $c$, many counter-examples are now known where eq. (1.1) is not satisfied $[5,6]$. Further, the latter investigations [6] also demonstrated that any linear combination of $c$ and $a$ will not generically satisfy eq. (1.1). This leaves us only to consider the central charge $a$.

It was Cardy [7] who originally conjectured that $a$ should decrease monotonically along RG flows of four-dimensional QFT's. Numerous nontrivial examples have been found supporting this conjecture, including perturbative fixed points [8] and supersymmetric gauge theories [6,9]. While a counter-example to Cardy's conjecture in $d=4$ was proposed in [10], recently, a certain flaw in this analysis was identified and so this possible counter-example is removed [11]. Further, as we review below, support for such a c-theorem in $d=4$ was found with the AdS/CFT correspondence $[12,13]$ - 
however, in the class of holographic theories studied there, $c=a$ and so both $a$ and $c$ satisfy eq. (1.1). While Cardy's conjecture is supported by numerous nontrivial examples, a general proof is still lacking. A primary purpose of this paper is to report further evidence for the conjecture coming from a broader class of holographic models, where in particular the central charges $a$ and $c$ are distinct. An preliminary report of these results was given in [14].

Cardy's conjecture [7] actually referred to any even number of spacetime dimensions. For even $d$, the trace anomaly for CFT's in a curved background can be written as $[2]$

$$
\left\langle T_{a}^{a}\right\rangle=\sum B_{i} I_{i}-2(-)^{d / 2} A E_{d}+B^{\prime} \nabla_{a} J^{a}
$$

where $E_{d}$ is the Euler density in $d$ dimensions and $I_{i}$ are the independent Weyl invariants of weight $-d .^{1}$ Finally the last term is a conformally invariant but also schemedependent total derivative. That is, this last contribution can be changed or even eliminated by adding a (finite) covariant counter-term to the action. Cardy's proposal is then that, in any even $d$, for RG flows connecting two fixed points

$$
(A)_{\mathrm{UV}} \geq(A)_{\mathrm{IR}}
$$

Of course, this coincides with Zamolodchikov's result in $d=2$ where $A=c / 12$ and it matches the above discussion for $d=4$ where $A=a$ with our present choice of normalization. ${ }^{1,2}$

One of the advantages of the investigating $\mathrm{RG}$ flows in a holographic framework is that the results are readily extended to arbitrary dimensions [12, 14]. In [14] and in the following, we examine holographic models with higher curvature gravity in the $(d+1)$-dimensional bulk, which allows us to distinguish the central charges appearing in the trace anomaly (1.4) of the $d$-dimensional boundary CFT's for even $d$. Hence we are able to discriminate between the behaviour of the various central charges in $\mathrm{RG}$ flows and we find that only $A$ has a natural monotonic flow, giving further support for Cardy's conjecture (1.5). Our analysis of holographic RG flows applies in arbitrary higher $d$ and in fact, we find a certain quantity, denoted $a_{d}^{*}$, satisfying an inequality such as that given in eq. (1.1) or (1.5) for any $d$, that is, for both even and odd $d$. There is no trace anomaly for odd $d$ and so some new interpretation for $a_{d}^{*}$ must be found in

\footnotetext{
${ }^{1}$ A note on our conventions: The stress tensor is defined by $T_{a b} \equiv-2 / \sqrt{-g} \delta I / \delta g^{a b}$. The Euler density $E_{d}$ is normalized so that on a $d$-dimensional sphere: $\oint_{S^{d}} d^{d} x \sqrt{g} E_{d}=2$. The Weyl invariants $I_{i}$ are constructed from contractions of $d / 2$ curvatures or $d / 2-k$ curvatures and $2 k$ covariant derivatives. There is some ambiguity in the construction of the $I_{i}$ which we (partially) fix by demanding that these invariants vanish when evaluated on a round $d$-dimensional sphere, i.e., $\left.I_{i}\right|_{S^{d}}=0$.

${ }^{2}$ Further, in comparing eqs. (1.3) and (1.4), we see for $d=4$ there is a single invariant $I_{1}$ corresponding to the Weyl tensor squared and for which $c=16 \pi^{2} B_{1}$.
} 
this case. Following [14], we identify this quantity with the coefficient of a universal contribution to the entanglement entropy for a particular construction for both odd and even $d$. Our results suggest that these coefficients appearing in entanglement entropy play the role of central charges for odd-dimensional CFT's and allow us to conjecture a c-theorem on the space of odd-dimensional field theories. However, we must emphasize that our higher curvature gravity actions are not derived from string theory. These theories should be regarded as toy models which allow us to explore of the role of higher curvature terms in holography. Ultimately, one would like to develop a better understanding of string theory in order study interesting holographic backgrounds with high curvatures and study the possibility of a holographic c-theorem in this framework.

An overview of the paper is as follows: We begin with a review of holographic c-theorem for Einstein gravity $[12,13]$ in section 2. Further, we describe a similar c-theorem for a particular higher curvature theory, known as quasi-topological gravity $[15,16]$. In section 3 , we extend our discussion to gravity theories with more general higher curvature interactions. We propose that the couplings of these new interactions should be tuned to remove any non-unitary operators from the dual boundary theory. With this constraint, we find that the resulting theories automatically satisfy a holographic c-theorem. In both of these sections, we are able to show that for even $d$, the quantity $a_{d}^{*}$ which obeys the holographic c-theorem is precisely the central charge $A$ in eq. (1.4). In section 4 , we demonstrate that $a_{d}^{*}$ appears in a certain calculation of entanglement entropy for odd or even $d$. In particular, we place the $d$-dimensional CFT on $S^{d-1} \times R$ and calculating the entanglement entropy of the ground state between two halves of the sphere. We then find a universal contribution: $S_{\text {univ }} \propto a_{d}^{*}$. In this section, the entanglement entropy is calculated by relating it to the thermal entropy of the CFT on the hyperbolic plane, i.e., $H^{d-1} \times R$, with a particular temperature. In section 5 , we describe a more conventional calculation of the entanglement entropy in the boundary CFT using the replica trick. The latter is translated to a holographic calculation in the bulk gravity theory and we reproduce the same results as in the previous section. Here we note that these calculations are distinct from the standard holographic calculations of entanglement entropy $[17,18]$, which are only applicable with the bulk theory is Einstein gravity. In section 5.4, we also extend our calculation using the replica trick to any CFT in even dimensions and show (without holography) that in general the universal coefficient is precisely the central charge $A$. Returning to the holographic framework in section 5.5, we show that $a_{d}^{*}$ can be thought of as counting the degrees of freedom in the boundary CFT. In section 6, we compare our results with two other proposals for charges which may satisfy a c-theorem in higher dimensions. We explicitly show that $a_{d}^{*}$ does not correspond to the coefficient governing the leading singularity of the two-point function of the stress tensor or the leading coefficient in the entropy density 
of a thermal bath. Further, we show that the latter coefficient need not satisfy a holographic c-theorem within the class of boundary theories described by quasi-topological gravity. We conclude with a discussion of our results and possible future directions in section 7 . We also have some appendices containing related calculations. In appendix A, we examine the holographic RG flows more generally for the theories considered in section 2. Appendix B makes some preliminary comments on establishing a holographic c-theorem when the matter fields couple to the higher curvature interactions. Finally in appendix C, we make some brief comments about possible c-theorems for holographic models with the non-relativistic Schrödinger symmetry.

\section{Holographic c-theorems - Take One}

The c-theorem was first considered in the context of the AdS/CFT correspondence by $[12,13]$. There one begins with $(d+1)$-dimensional Einstein gravity coupled to various matter fields:

$$
I=\frac{1}{2 \ell_{\mathrm{P}}^{d-1}} \int d^{d+1} x \sqrt{-g}\left(R+\mathcal{L}_{\text {matter }}\right)
$$

The matter theory is assumed to have various stationary points where $\mathcal{L}_{\text {matter }}=$ $d(d-1) \alpha_{i} / L^{2}$ with some canonical scale $L$. While the latter is phrased in a general way, it is useful to keep in mind a simple example in the following. Namely, the matter sector here could naturally be a scalar field theory where the potential has a number of extrema with $V_{i, c r i t}=-d(d-1) \alpha_{i} / L^{2}$. The vacuum energy or cosmological constant is negative at all of the relevant stationary points and it is a convenient notation to introduce $\alpha_{i}$ to distinguish the different values. At these points, the vacuum solution for the Einstein theory is simply $\operatorname{AdS}_{d+1}$ with the curvature scale given by $\tilde{L}^{2}=L^{2} / \alpha_{i}$.

Next one considers solutions of the above theory (2.1) where the scalar sits at one fixed point in the asymptotic (UV) region and makes a smooth transition to another fixed point in the interior (IR) region. Such a solution can be interpreted as a holographic representation of a renormalization group flow, in which the boundary CFT flows from one fixed point in the UV to another in the IR. The spacetime geometry for these holographic RG flows is conveniently described with a metric of the form

$$
d s^{2}=e^{2 A(r)}\left(-d t^{2}+d \vec{x}_{d-1}^{2}\right)+d r^{2} .
$$

This metric becomes that for $\operatorname{AdS}_{d+1}$ with $A(r)=r / \tilde{L}$ at the stationary points. Now one defines [12]:

$$
a(r) \equiv \frac{\pi^{d / 2}}{\Gamma(d / 2)\left(\ell_{\mathrm{P}} A^{\prime}(r)\right)^{d-1}},
$$


where 'prime' denotes a derivative with respect to $r$. Then for general solutions with the above metric (2.2), one finds

$$
\begin{aligned}
a^{\prime}(r) & =-\frac{(d-1) \pi^{d / 2}}{\Gamma(d / 2) \ell_{\mathrm{P}}^{d-1} A^{\prime}(r)^{d}} A^{\prime \prime}(r) \\
& =-\frac{\pi^{d / 2}}{\Gamma(d / 2) \ell_{\mathrm{P}}^{d-1} A^{\prime}(r)^{d}}\left(T^{t}{ }_{t}-T_{r}^{r}\right) \geq 0 .
\end{aligned}
$$

In the second equality above, the Einstein equations are used to eliminate $A^{\prime \prime}(r)$ in favour of components of the stress tensor. The final inequality assumes that the matter fields obey the null energy condition [19]. Combining this monotonic evolution of $a(r)$ with $r$ with the standard connection between $r$ and energy scale in the CFT, $a(r)$ always decreases in flowing from the UV (large $r$ ) to the IR ( $\operatorname{small} r$ ).

To make a more precise interpretation of the bulk solutions in terms of the boundary CFT, it is simplest to focus the discussion on $d=4$ at this point. In this case, the holographic trace anomaly [20] allows one to calculate the two central charges, $a$ and $c$, of the four-dimensional CFT - see eq. (1.3). For any of the $\mathrm{AdS}_{5}$ vacua with a curvature scale $\tilde{L}$, one finds

$$
c=a \quad \text { and } \quad a=\pi^{2} \frac{\tilde{L}^{3}}{\ell_{\mathrm{P}}^{3}}=\left.a(r)\right|_{A d S} .
$$

As we emphasize below, the equality of the two central charges results because the bulk theory is Einstein gravity [20]. However, the important observation is that the value of the flow function (2.3) will precisely match that of the central charges in the dual CFT at each of the fixed points. Hence with the assumption of the null energy condition, the holographic CFT's dual to Einstein gravity (3.1) satisfy Cardy's proposed c-theorem. That is, for these holographic RG flows, $a$ is always larger at the UV fixed point than at the IR fixed point. Of course, these holographic models do not distinguish between the flow of $a$ and $c$, and so the central charge $c$ obeys the same inequality as well.

It has long been known that to construct a holographic model where $a \neq c$, the gravity action must include higher curvature interactions [21]. In part, this motivated the recent construction of the higher curvature theory, known as quasi-topological gravity [15]. This gravitational theory should be regarded as a toy model which allows us to explore the behaviour of a broader class of holographic CFT's. It was demonstrated in [14] that this bulk theory also naturally exhibits a holographic c-theorem, as follows: 
The action for quasi-topological gravity can be written as [15]

$$
\begin{gathered}
I=\frac{1}{2 \ell_{\mathrm{P}}^{d-1}} \int \mathrm{d}^{d+1} x \sqrt{-g}\left[\frac{d(d-1)}{L^{2}} \alpha+R+\frac{\lambda L^{2}}{(d-2)(d-3)} \mathcal{X}_{4}\right. \\
\left.-\frac{8(2 d-1) \mu L^{4}}{(d-5)(d-2)\left(3 d^{2}-21 d+4\right)} \mathcal{Z}_{d+1}\right]
\end{gathered}
$$

where $\mathcal{X}_{4}$ is the four-dimensional Euler density, as used in Gauss-Bonnet gravity [22],

$$
\mathcal{X}_{4}=R_{a b c d} R^{a b c d}-4 R_{a b} R^{a b}+R^{2}
$$

and $\mathcal{Z}_{d+1}$ is the new curvature-cubed interaction $[15,23]$

$$
\begin{aligned}
& \mathcal{Z}_{d+1}=R_{a b}^{c}{ }^{d} R_{c d}{ }^{f}{ }^{f} R_{e}{ }^{a}{ }^{b}+\frac{1}{(2 d-1)(d-3)}\left(\frac{3(3 d-5)}{8} R_{a b c d} R^{a b c d} R\right. \\
& -3(d-1) R_{a b c d} R_{e}^{a b c} R^{d e}+3(d-1) R_{a b c d} R^{a c} R^{b d} \\
& \left.+6(d-1) R_{a}{ }^{b} R_{b}{ }^{c} R_{c}{ }^{a}-\frac{3(3 d-1)}{2} R_{a}{ }^{b} R_{b}{ }^{a} R+\frac{3(d-1)}{8} R^{3}\right) .
\end{aligned}
$$

This action is written for any (boundary) dimension $d \geq 4$, although we set $\mu=0$ for $d=5$ to avoid the singular behaviour of the pre-factor of $\mathcal{Z}_{d+1}$ in eq. (2.6). By introducing interactions quadratic and cubic in the curvature, this holographic model allows one to explore the full three-parameter space of coefficients controlling the twoand three-point functions of the stress tensor in a general $d$-dimensional CFT [24]. The reader should keep in mind that this action (2.6) was not derived from string theory. Rather, as noted above, it was constructed as a toy model to allow us to explore a broader class of holographic CFT's while maintaining control within the gravity calculations. However, we should also note that the gravitational couplings, $\lambda$ and $\mu$, in eq. (2.6) are constrained to be not very large, otherwise one finds that the dual CFT is inconsistent - for a more precise discussion, see [16, 25, 26, 27]. We emphasize that the discussions here and in $[15,16]$ should only be regarded as an initial exploration of the role of higher curvature terms in holography. Ultimately, one would like to develop our understanding of string theory to the point where we can study interesting holographic backgrounds with high curvatures.

We have also introduced a factor of $\alpha(>0)$ in the cosmological constant term above in anticipation of our consideration of holographic RG flows below. The idea is that as in eq. (2.6), the gravity theory is coupled to a standard matter theory, e.g., a scalar field, with various stationary points which yield different values for the parameter $\alpha$. At any of these stationary points, there is an $\mathrm{AdS}_{d+1}$ solution with a curvature scale $\tilde{L}^{2}=L^{2} / f_{\infty}$ where

$$
\alpha=f_{\infty}-\lambda f_{\infty}^{2}-\mu f_{\infty}^{2}
$$


In general, this equation yields three roots for $f_{\infty}$. However, for any choice of the couplings $\lambda$ and $\mu$, at most one of these roots corresponds to a ghost-free AdS vacuum which supports nonsingular black hole solutions, as described in detail in [15]. Further, in the case that the couplings, $\lambda$ and $\mu$, are not large, this will be the root that is continuously connected to the single root (i.e., $f_{\infty}=\alpha$ ) that remains in the limit $\lambda, \mu \rightarrow 0$. Implicitly, we will be working in this regime of the coupling space and with this particular root in the following.

Originally, this action (2.6) was constructed to give a theory for which AdS black hole solutions could be easily found analytically [15]. However, another remarkable property of quasi-topological gravity is that the linearized graviton equations in the $\mathrm{AdS}_{d+1}$ vacuum are only second order in derivatives [15]. In fact, up to an overall numerical factor, the linearized equations are precisely the same as those for Einstein gravity in the $\operatorname{AdS}_{d+1}$ background. If we focus on $d=4$ for a moment, the techniques of [20] can be applied to calculate the central charges [16]

$$
\begin{aligned}
& c=\pi^{2} \frac{\tilde{L}^{3}}{\ell_{\mathrm{P}}^{3}}\left(1-2 \lambda f_{\infty}-3 \mu f_{\infty}^{2}\right), \\
& a=\pi^{2} \frac{\tilde{L}^{3}}{\ell_{\mathrm{P}}^{3}}\left(1-6 \lambda f_{\infty}+9 \mu f_{\infty}^{2}\right) .
\end{aligned}
$$

These expressions make clear that $a \neq c$ as long as the higher curvature couplings are nonvanishing.

Now to examine holographic RG flows in quasi-topological gravity (for arbitrary $d$ ), we adopt the same metric ansatz (2.2). As above, at a stationary point of the matter sector with a fixed $\alpha$, the $A d S_{d+1}$ vacua again correspond to $A(r)=r / \tilde{L}$. In this case, we construct a new flow function as [14]

$$
\begin{aligned}
a_{d}(r) \equiv & \frac{\pi^{d / 2}}{\Gamma(d / 2)\left(\ell_{\mathrm{P}} A^{\prime}(r)\right)^{d-1}} \\
& \times\left(1-\frac{2(d-1)}{d-3} \lambda L^{2} A^{\prime}(r)^{2}-\frac{3(d-1)}{d-5} \mu L^{4} A^{\prime}(r)^{4}\right) .
\end{aligned}
$$

Now examining the radial evolution of $a_{d}(r)$, we find

$$
\begin{aligned}
a^{\prime}(r) & =-\frac{(d-1) \pi^{d / 2}}{\Gamma(d / 2) \ell_{\mathrm{P}}^{d-1} A^{\prime}(r)^{d}} A^{\prime \prime}(r)\left(1-2 \lambda L^{2} A^{\prime}(r)^{2}-3 \mu L^{4} A^{\prime}(r)^{4}\right) \\
& =-\frac{\pi^{d / 2}}{\Gamma(d / 2) \ell_{\mathrm{P}}^{d-1} A^{\prime}(r)^{d}}\left(T^{t}{ }_{t}-T^{r}{ }_{r}\right) \geq 0 .
\end{aligned}
$$

Again, the gravitational equations of motion allow us to introduce the components of the stress tensor in going from the first to second line. Further, as above, we also assume 
the null energy condition for the final inequality to hold. We apply this constraint here in the spirit of constructing a toy model with reasonable physical properties. One might note that violations of the null energy condition have been argued to lead to instabilities quite generally [28]. In the context of a full string theory or theory of quantum gravity, we expect that this condition will be relaxed but to make progress here, we assume that the matter sector continues to obey the null energy condition as a pragmatic choice - see section 7 for further discussion. With the latter assumption then, $a(r)$ evolves monotonically along the holographic RG flows and we can conclude that the corresponding 'central charge' is always larger in the UV than at the IR fixed point.

Note that there is a technical point which we must address for odd $d$. In this case, it could be that the expression in the second line of eq. (2.13) is negative if $A^{\prime}(r)<0$. However, we can rule out this possibility as follows: By construction, our flow geometry will have an asymptotically $\mathrm{AdS}_{d+1}$ region at large $r$ where $A^{\prime}(r)>0$. Now imagine that in the interior, $A^{\prime}(r)$ is negative over some region $r_{0}<r<r_{1}$ and positive from $r_{1}$ out to the asymptotic boundary. Hence at the radius $r_{1}$, we must have had $A^{\prime}\left(r_{1}\right)=0$ and $A^{\prime \prime}\left(r_{1}\right)>0$. However, this leads to a contradiction. If we evaluate the equation of motion $(d-1) A^{\prime \prime}(r)\left(1-2 \lambda L^{2} A^{\prime}(r)^{2}-3 \mu L^{4} A^{\prime}(r)^{4}\right)=T_{t}^{t}-T_{r}^{r}$ at $r=r_{1}$ and combine this result with the null energy condition, we find $A^{\prime \prime}\left(r_{1}\right) \leq 0$. Hence our assumption that there is some region where $A^{\prime}(r)<0$ must be incorrect. ${ }^{3}$

Let us denote the fixed point value of the flow function (2.12) as

$$
\left.a_{d}^{*} \equiv a_{d}(r)\right|_{A d S}=\frac{\pi^{d / 2} \tilde{L}^{d-1}}{\Gamma(d / 2) \ell_{\mathrm{P}}^{d-1}}\left(1-\frac{2(d-1)}{d-3} \lambda f_{\infty}-\frac{3(d-1)}{d-5} \mu f_{\infty}^{2}\right)
$$

Then with eq. (2.13), our holographic model satisfies a holographic c-theorem which specifies that

$$
\left(a_{d}^{*}\right)_{U V} \geq\left(a_{d}^{*}\right)_{I R}
$$

Having found that $a_{d}^{*}$ satisfies a c-theorem, one is left to determine what this quantity corresponds to in the dual CFT. Inserting $d=4$ into eq. (2.14) and comparing with eq. (2.11), we see that $a_{4}^{*}$ is precisely the central charge $a$. Motivated by Cardy's conjecture (1.5) for a c-theorem in QFT's in even dimensional spacetimes, it is natural to compare $a_{d}^{*}$ to the coefficient $A$ in eq. (1.4). In fact, using the approach of $[29],{ }^{4}$ one

\footnotetext{
${ }^{3}$ In the special case that $A^{\prime \prime}\left(r_{1}\right)=0$, we can assume that it can be expressed in terms of a Taylor expansion around $r_{1}$. To leading order, we would have $A^{\prime \prime}(r) \simeq k\left(r-r_{1}\right)^{n}$ with $k>0$ and $n$ is some positive and even integer. Then, as above, we again find a contradiction in the vicinity around $r=r_{1}$ and reach the same conclusion.

${ }^{4}$ See discussion around eq. (5.23) for more details of this calculation.
} 
readily confirms that there is again a precise match

$$
a_{d}^{*}=A \quad \text { for even } d .
$$

Hence again, we find support for Cardy's conjecture with this broad class of holographic CFT's. However, we must seek a broader definition of $a_{d}^{*}$ in order to understand our results for odd $d$. We address this question in sections 4 and 5 , where we show that $a_{d}^{*}$ emerges in a certain calculation of entanglement entropy.

\section{Holographic c-theorems - Take Two}

In section 2, we have shown that quasi-topological gravity naturally gives rise to a holographic c-theorem. Further at the fixed points, we identified the quantity that decreases along the RG flows as the coefficient of the A-type trace anomaly of the dual conformal field theory, for even $d$. Now we would like to test how robust this result is by expanding our considerations of holographic RG flows to a broader class of gravitational theories. In the following, we begin with a completely general curvaturecubed action and develop a series of constraints so that the resulting holographic model is physically reasonable. Again, our analysis here should be considered as an exploration of holography with certain toy models which display credible physical properties.

To begin, we will consider an action of the form

$$
I=\frac{1}{2 \ell_{\mathrm{P}}^{d-1}} \int \mathrm{d}^{d+1} x \sqrt{-g}\left[\frac{d(d-1)}{L^{2}} \alpha+R+L^{2} \widetilde{\mathcal{X}}+L^{4} \widetilde{\mathcal{Z}}\right]
$$

where $\mathcal{X}$ and $\mathcal{Z}$ contain general interactions quadratic and cubic in the curvature

$$
\begin{aligned}
\widetilde{\mathcal{X}} & =\lambda_{1} R_{a b c d} R^{a b c d}+\lambda_{2} R_{a b} R^{a b}+\lambda_{3} R^{2} \\
\widetilde{\mathcal{Z}} & =\mu_{1} R_{a b}^{c d} R_{c d}^{e f} R_{e f}^{a b}+\mu_{2} R_{a b}{ }^{c d} R_{c d}{ }^{e f} R_{e f}{ }^{a b}+\mu_{3} R_{a b c d} R^{a b c} R^{d e} \\
& \quad+\mu_{4} R_{a b c d} R^{a b c d} R+\mu_{5} R_{a b c d} R^{a c} R^{b d}+\mu_{6} R_{a}^{b} R_{b}^{c} R_{c}^{a}+\mu_{7} R_{a}^{b} R_{b}^{a} R+\mu_{8} R^{3} .
\end{aligned}
$$

In constructing $\widetilde{\mathcal{Z}}$, we began with all possible six-derivative interactions and eliminated terms which are redundant because of index symmetries or the Bianchi identities or which are total derivatives [15]. In fact, this construction yields two additional independent terms, $\nabla_{a} R_{b c} \nabla^{a} R^{b c}$ and $\nabla_{a} R \nabla^{a} R$. However, we have discarded these terms in eq. (3.3) for simplicity as we would find that the corresponding coefficients are always set to zero with the constraints introduced in the following discussion.

We again assume that there is a matter sector, which exhibits stationary points with different values of $\alpha$ in the cosmological constant term in eq. (3.1). At any of these critical points, there is an $\operatorname{AdS}_{d+1}$ with a curvature scale $\tilde{L}^{2}=L^{2} / f_{\infty}$ where

$$
\alpha=f_{\infty}-\hat{\lambda} f_{\infty}^{2}-\hat{\mu} f_{\infty}^{2},
$$


with

$$
\begin{aligned}
\hat{\lambda}= & \frac{d-3}{d-1}\left(2 \lambda_{1}+d \lambda_{2}+d(d+1) \lambda_{3}\right) \\
\hat{\mu}=-\frac{d-5}{d-1}( & (d-1) \mu_{1}+4 \mu_{2}+2 d \mu_{3}+2 d(d+1) \mu_{4} \\
& \left.\quad+d^{2} \mu_{5}+d^{2} \mu_{6}+d^{2}(d+1) \mu_{7}+d^{2}(d+1)^{2} \mu_{8}\right) .
\end{aligned}
$$

Of course, we have arranged eq. (3.4) to take the same form as eq. (2.9) in the previous section. In general, this cubic equation again yields three roots for $f_{\infty}$. However, as in the previous section, when the couplings, $\lambda_{i}$ and $\mu_{i}$, are not large, there will be one root that is continuously connected to the single root (i.e., $f_{\infty}=\alpha$ ) that remains in the limit of Einstein gravity, i.e., $\lambda_{i}, \mu_{i} \rightarrow 0$. Implicitly, we will be working in this regime in the following and $f_{\infty}$ will refer to this particular root. We have not analyzed the general theory in great detail but we expect that, as for quasi-topological gravity, the vacua corresponding to any other (real) roots will be problematic [15].

While we could examine holographic RG flows with this action with general curvaturesquared and -cubed interactions, it seems unreasonable to expect that any such arbitrary gravity theory should yield a holographic c-theorem, just as it is unreasonable to expect that any arbitrary quantum field theories should satisfy a c-theorem. In particular, we do not expect that non-unitary QFT's will satisfy a c-theorem. Hence we must ask how should we constrain the new couplings in this gravitational action (3.1) in order to produce a physically credible model. Quasi-topological gravity has a number of interesting properties which make it a reasonable toy model for holographic studies. One striking feature of the theory was that although the general equations of motion are fourth order in derivatives, if the equations of motion for gravitons propagating in the AdS vacuum are only second order [15]. While this feature greatly facilitates holographic investigations of this theory, as explicitly seen in [16], there is a deeper significance to this property, as we now discuss.

Given the general gravitational action (3.1), the full equations of motion will be fourth order in derivatives, as explicitly shown in [30]. Further even if considering the equations of motion for graviton propagation in a general background solution or in the AdS vacuum, these linearized equations are still fourth order. To gain some intuition for such higher order equations, we establish an analogy with a higher-derivative scalar field equation (in flat space) - following [16]. To begin, we would think of a simple massless scalar (i.e., $\square \phi=0$ ) as providing the analog of the linearized Einstein equations. Then we modify this equation with the addition of a fourth order term to model the graviton 
equations produced in our generalized gravity theory (3.1)

$$
\left(\square+\frac{a}{M^{2}} \square^{2}\right) \phi=0
$$

Here we imagine $M^{2}$ is some high energy scale (the analog of $1 / L^{2}$ ) and $a$ is the dimensionless coupling that controls the strength of the higher-derivative term (the analog of $\lambda_{i}$ and $\mu_{i}$ ). The (flat space) propagator for this scalar can now be written as

$$
\frac{1}{q^{2}\left(1-a q^{2} / M^{2}\right)}=\frac{1}{q^{2}}-\frac{1}{q^{2}-M^{2} / a} .
$$

Now the $1 / q^{2}$ pole is associated with the regular modes which are easily excited at low energies. The second pole $1 /\left(q^{2}-M^{2} / a\right)$ is associated with additional 'physical' modes that appear at the high energy scale. Depending on the sign of $a$, these new modes may have a regular mass $(a<0)$ or be tachyonic $(a>0)$. However, the key point is that these extra high energy modes are ghosts (for either sign of $a$ ) because the overall sign of their contribution to the propagator (3.7) is negative. This appearance of ghosts is a generic feature of higher derivative equations of motion and so one must worry that the fourth order graviton equations generically emerging from eq. (3.1) indicate that these gravitational theories contain ghosts. From a holographic perspective, this indicates that the graviton couples to more than the usual stress tensor in the boundary CFT. The massive ghost modes indicate that metric fluctuations also mixes with an additional tensor operator which is non-unitary. That is, the new operator produces states with negative norm in the CFT. Hence from either perspective, there is a fundamental pathology with such a theory.

However, as the analysis of quasi-topological gravity indicates [15], this problem can be evaded at least in the AdS vacuum. That is, we can tune the coupling constants in eq. (3.1) to special values, $\lambda_{i}^{*}$ and $\mu_{i}^{*}$, so that the linearized graviton equations in the AdS vacuum are only second order in derivatives. This tuning eliminates the appearance of ghosts in the gravity theory and of non-unitary operators in the boundary CFT. In the scalar field analogy above, specially tuned simply corresponds to $a=0$. However, note that as we approach $a=0$ from finite values, the mass of the ghost modes diverges. Hence in the context of our holographic model, we can understand that the non-unitary operators are removed from the spectrum of the boundary CFT because, as we adjust the higher curvature coupling constants to approach the ghostfree model, the conformal dimension of these operators diverges. Further note, that after we have fixed the couplings to $\lambda_{i}^{*}$ and $\mu_{i}^{*}$, we are able to calculate arbitrary $n$ point functions of the stress tensor in the vacuum of the boundary CFT, with the usual perturbative expansion in terms of Witten diagrams [31]. Hence to begin, we impose 
this requirement to constrain the gravitational action (3.1) as a tentative step towards producing a physically interesting holographic model. Afterwards, we will examine whether RG flows in these theories also obey a holographic c-theorem.

To identify the constraints leading to second order linearized equations of motion for fluctuations, we proceed as follows: First, we write the $\operatorname{AdS}_{d+1}$ metric as ${ }^{5}$

$$
d s^{2}=e^{2 r / \tilde{L}}\left(-d t^{2}+d \vec{x}_{d-1}^{2}\right)+d r^{2} .
$$

Next (using Mathematica), we consider the linearized equations of motion around this background, including all possible metric fluctuations $h_{\mu \nu} d x^{\mu} d x^{\nu}$ where $h_{\mu \nu}$ are allowed to depend on all coordinates. Isolating the coefficient of $\partial_{r}^{4} h_{12}$ in these equations, we find that this coefficient can be set to zero with

$4 \lambda_{1}+\lambda_{2}+f_{\infty}\left[3 \mu_{1}-24 \mu_{2}-4(d+1) \mu_{3}-4 d(d+1) \mu_{4}-(2 d-1) \mu_{5}-3 d \mu_{6}-d(d+1) \mu_{7}\right]=0$.

Then we look at the coefficient of $\partial_{r}^{4} h_{11}$ and set this to zero with

$$
\lambda_{1}-\lambda_{3}+\frac{f_{\infty}}{2}\left[3 \mu_{1}-12 \mu_{2}-2 d \mu_{3}-2\left(d^{2}+d-4\right) \mu_{4}+2 \mu_{5}+4 d \mu_{7}+6 d(d+1) \mu_{8}\right]=0 .
$$

Remarkably, one finds that this two constraints alone are sufficient to eliminate all of the higher order contributions to the linearized equations of motion! In the context of RG flows, $f_{\infty}$ will change between the various fixed points. As a result, it is prudent to demand that the above constraints hold for any value of $f_{\infty}{ }^{6}$ Thus we are led to the following constraints:

$$
\begin{aligned}
& \lambda_{2}=-4 \lambda_{1}, \quad \lambda_{3}=\lambda_{1}, \\
& \mu_{7}= \frac{1}{d(d+1)}\left(3 \mu_{1}-24 \mu_{2}-4(d+1) \mu_{3}-4 d(d+1) \mu_{4}-(2 d-1) \mu_{5}-3 d \mu_{6}\right)(31 \\
& \mu_{8}=\frac{1}{d(d+1)}\left(-\frac{d+5}{2(d+1)} \mu_{1}+\frac{2(d+9)}{d+1} \mu_{2}+\frac{d+8}{3} \mu_{3}\right. \\
&\left.\quad+\frac{1}{3}\left(d^{2}+9 d-4\right) \mu_{4}+\frac{d-1}{d+1} \mu_{5}+\frac{2 d}{d+1} \mu_{6}\right)
\end{aligned}
$$

While eq. (3.12) follows directly from eq. (3.9), the constraint in eq. (3.13) comes from taking a linear combination of the expressions appearing in both eqs. (3.9) and (3.10). Note that the conditions (3.11) on the $\lambda_{i}$ yield the Gauss-Bonnet combination of curvature-squared interactions [22], as expected, i.e., $\left(\lambda_{1}, \lambda_{2}, \lambda_{3}\right) \propto(1,-4,1)$.

\footnotetext{
${ }^{5}$ We will assume $d \geq 3$. The $d=2$ case has been considered in [32, 33, 30, 34].

${ }^{6}$ Note that this condition cannot be satisfied when $d=2$, as explained in [30].
} 
With the constraints (3.11-3.13) above, we have ensured that we have a reasonable (i.e., unitary) boundary theory for the $\mathrm{AdS}_{d+1}$ vacua. Hence we might examine if these theories satisfy a holographic c-theorem. So following the experience developed in the previous section, we substitute in the RG flow geometry (2.2) and examine the gravitational equation of motion proportional to $T^{t}{ }_{t}-T_{r}^{r}$. However, unfortunately, the resulting equation as terms proportional to the third and fourth derivative of the conformal factor, i.e., $A^{\prime \prime \prime}$ and $A^{\prime \prime \prime \prime}$. In order to get a simple c-theorem as in the previous section, we can eliminate these terms by fixing

$$
\begin{gathered}
\mu_{6}=\frac{1}{(d-1)^{3}}\left(-2(d-3) \mu_{1}+8(3 d-5) \mu_{2}+\frac{2}{3}(d+1)(5 d-9) \mu_{3}\right. \\
\left.+\frac{16}{3} d(d+1)(d-2) \mu_{4}+2(d-1)^{2} \mu_{5}\right) .
\end{gathered}
$$

Of course, with hindsight, the interpretation of this problem is obvious. We have ensured that the non-unitary operators corresponding to the ghost-like graviton modes have been removed from the CFT spectrum at any fixed points. However, when the boundary theory is perturbed away from the fixed points, the non-unitary operator come in from infinity to 'pollute' the RG flow. The solution is then also obvious. We should demand that the linearized equations of motion for any fluctuations around the RG flow geometry (2.2) are second order in derivatives. This will ensure that the boundary QFT does not contain any non-unitary operators along the RG flows, as well as at the fixed points.

As before we considering general fluctuations around the RG flow metric (2.2), we examine higher order contributions to the linearized equations of motion. If we have already imposed eqs. (3.11-3.13), the coefficients of three new terms proportional to $A^{\prime \prime} \partial_{r}^{4} h_{11}, A^{\prime \prime} \partial_{r}^{4} h_{12}$ and $A^{\prime \prime} \partial_{t}^{4} h_{12}$ can be set to zero with eq. (3.14) and

$$
\begin{aligned}
& \mu_{4}=\frac{1}{8 d}\left(3 \mu_{1}-12 \mu_{2}-(d+3) \mu_{3}\right), \\
& \mu_{5}=-\frac{1}{d-1}\left(12 \mu_{2}+(d+1) \mu_{3}\right) .
\end{aligned}
$$

The seven constraints in eqs. (3.11-3.16) are necessary and sufficient to produce to two-derivative equations for metric fluctuations around a general RG flow (2.2). This seems like the best approach to constructing a holographic model with a physically reasonable boundary theory in the present study of $\mathrm{RG}$ flows.

As noted above with eq. (3.14), the above constraints also ensure that, with the RG flow metric (2.2), the gravitational equation of proportional to $T^{t}{ }_{t}-T^{r}{ }_{r}$ takes the 
same simple form found in the previous section. Hence we construct the flow function

$$
\begin{aligned}
a_{d}(r) \equiv & \frac{\pi^{d / 2}}{\Gamma(d / 2)\left(\ell_{\mathrm{P}} A^{\prime}(r)\right)^{d-1}} \\
& \times\left(1-\frac{2(d-1)}{d-3} \hat{\lambda} L^{2} A^{\prime}(r)^{2}-\frac{3(d-1)}{d-5} \hat{\mu} L^{4} A^{\prime}(r)^{4}\right) .
\end{aligned}
$$

Here, with the constraints (3.11-3.16), the couplings defined in eq. (3.5) reduce to

$$
\begin{aligned}
& \hat{\lambda}=(d-3)(d-2) \lambda_{1}, \\
& \hat{\mu}=\frac{(d-5)(d-3)(d-2)}{24}\left(3 \mu_{1}-12 \mu_{2}-(d-1) \mu_{3}\right) .
\end{aligned}
$$

Now by construction the radial derivative of $a_{d}(r)$ yields

$$
a^{\prime}(r)=-\frac{\pi^{d / 2}}{\Gamma(d / 2) \ell_{\mathrm{P}}^{d-1} A^{\prime}(r)^{d}}\left(T^{t}{ }_{t}-T_{r}^{r}\right) \geq 0 .
$$

As before, we again assume the null energy condition holds to produce the final inequality. If, as before, we denote the fixed point value of the flow function (3.17) as

$$
\left.a_{d}^{*} \equiv a_{d}(r)\right|_{A d S}=\frac{\pi^{d / 2} \tilde{L}^{d-1}}{\Gamma(d / 2) \ell_{\mathrm{P}}^{d-1}}\left(1-\frac{2(d-1)}{d-3} \hat{\lambda} f_{\infty}-\frac{3(d-1)}{d-5} \hat{\mu} f_{\infty}^{2}\right),
$$

then our higher curvature theories satisfy the holographic c-theorem

$$
\left(a_{d}^{*}\right)_{U V} \geq\left(a_{d}^{*}\right)_{I R}
$$

Hence we are led to conclude that demanding unitarity of the boundary theory along the $R G$ flows also guarantees that the theory obeys a holographic c-theorem.

In fact, the unitarity constraints, (3.11-3.16), are more than sufficient to produce a holographic c-theorem. If we only require that the $T^{t}{ }_{t}-T_{r}{ }_{r}$ equation is second order in derivatives and that a holographic c-theorem arises, the necessary constraints can be written as

$$
\begin{array}{r}
\lambda_{1}+\frac{d+1}{4} \lambda_{2}+d \lambda_{3}=0, \\
3 \mu_{1}+2 \mu_{3}+4 d \mu_{4}+(2 d+1) \mu_{5}+3 \mu_{6}+d(d+5) \mu_{7}+12 d^{2} \mu_{8}=0, \\
4 \mu_{2}+(d+1) \mu_{3}+4 d \mu_{4}+d \mu_{5}+\frac{d^{2}+1}{2} \mu_{6}+d(d+1) \mu_{7}+4 d^{2} \mu_{8}=0 .
\end{array}
$$

Considering the curvature-squared couplings $\lambda_{i}$, it is easy to see that the solution of eq. (3.11) also satisfies eq. (3.22) above. However, eq. (3.22) admits a two parameter 
space of solutions and so the unitary solution is only a special case within this larger set. Clearly, analogous comments apply for the curvature-cubed couplings $\mu_{i}$. However, the conclusion seems to be that some 'unphysical' models with nonunitary operators still satisfy a holographic c-theorem. It is perhaps not too surprising that such circumstances can arise since the RG flows only probe a small part of the full boundary theory. What is more important is that all of the holographic models with a unitary boundary theory are guaranteed to satisfy a holographic c-theorem.

In fact, the above discussion is incomplete for the constraints needed to ensure that the boundary theory is unitary. Having imposed the above constraints, the quadratic action for gravitons in the $\mathrm{AdS}_{d+1}$ vacua takes the form of a Fierz-Pauli action

$$
\begin{array}{rl}
S=\frac{\tilde{c}}{2 \ell_{\mathrm{P}}^{d-1}} \int d^{d+1} & x \sqrt{-g}\left(\frac{1}{4} \nabla_{\mu} h_{\rho \lambda} \nabla^{\mu} h^{\rho \lambda}-\frac{1}{2} \nabla_{\mu} h_{\rho \lambda} \nabla^{\rho} h^{\mu \lambda}+\frac{1}{2} \nabla_{\mu} h^{\mu \nu} \nabla_{\nu} h\right. \\
& \left.-\frac{1}{4} \nabla_{\mu} h \nabla^{\mu} h-\frac{d(d-1)}{2 \tilde{L}^{2}}\left(h^{\mu \nu} h_{\mu \nu}-\frac{1}{2} h^{2}\right)+O\left(h^{3}\right)\right) .
\end{array}
$$

where the constant pre-factor is given by $\tilde{c}=1-2 \hat{\lambda} f_{\infty}-3 \hat{\mu} f_{\infty}^{2}$. As we will see in section 6 , this coefficient controls the strength of the leading singularity in the two-point function of the stress tensor in the boundary CFT. In order to avoid ghost-like gravitons and to have a unitary boundary theory, we should also impose $\tilde{c}>0$. However, it is straightforward to see that this constraint is always satisfied because of our assumption about which root of eq. (3.4) we are considering - recall the discussion below eq. (3.5). We begin by denoting the right-hand side of eq. (3.4) as $h(f)=f-\hat{\lambda} f^{2}-\hat{\mu} f^{3}$. Now we are choosing $f_{\infty}$ to be the smallest positive root of the equation $h(f)=\alpha$. Note that since $h(f=0)=0$ and $\alpha>0$, the function must have a positive slope at this root, i.e., $h^{\prime}\left(f=f_{\infty}\right)>0$. However, recognizing that our expression above is precisely $\tilde{c}=h^{\prime}\left(f=f_{\infty}\right)$, we have established that $\tilde{c}>0$ for this root. ${ }^{7}$

There is a technical issue for $d=3$ and 5 , which we now briefly address. Let us focus on the case $d=3$ to be specific. Analogous comments will apply for $d=5$ and we return to this case below. If we examine eq. (3.17), it seems that our construction is singular with $d=3$. However, this is a spurious singularity as the factor of $1 /(d-3)$ in the second term can be absorbed into our definition of $\hat{\lambda}$ in eq. (3.18). More importantly, this contribution to $a_{d}(r)$ is proportional to $A^{\prime}(r)^{2} / A^{\prime}(r)^{d-1}$ and so is simply a constant in $d=3$. This is related to an obvious ambiguity in constructing the flow function, namely, we can always add a constant to $a_{d}(r)$, which, of course, does not effect the radial evolution. While in general there is no motivation to add an extra constant, it

\footnotetext{
${ }^{7}$ One might be concerned that we could find $h^{\prime}\left(f=f_{\infty}\right)=0$ but this only occurs outside of our chosen domain with $\lambda_{i}$ and $\mu_{i}$ 'not large' - see a full discussion for quasi-topological gravity in [15].
} 
turns out that there is a natural choice to make here. Quite generally, we will find that the flow function is proportional to a Wald-type formula [35]

$$
\frac{\partial \mathcal{L}}{\partial R^{t r} t r} \propto A^{\prime}(r)^{d-1} a_{d}(r)
$$

For further discussion of this relation, we refer the reader to sections 4 and 5, as well as refs. $[32,30]$. In any event, if we want to preserve this relation for $d=3$, we should add a constant term to the flow function as follows

$$
a_{3}(r)=\frac{\pi^{2}}{\ell_{\mathrm{P}}^{2} A^{\prime}(r)^{2}}\left(1-4 \lambda_{1} L^{2} A^{\prime}(r)^{2}+3 \hat{\mu} L^{4} A^{\prime}(r)^{4}\right) .
$$

The appearance of this constant above is related to the fact that, in the gravity action (3.1) with the constraint (3.11), the curvature-squared terms are proportional to the four-dimensional Euler density (2.7). The latter does not effect the gravitational equations of motion since the bulk theory is four-dimensional with $d=3$ but it still contributes to black hole entropy [36]. ${ }^{8}$ Now returning to $d=5$, analogous comments apply but it is now the contribution proportional to $A^{\prime}(r)^{4} / A^{\prime}(r)^{d-1}$ which is a constant. We again fix this contribution through eq. (3.26) to produce

$$
a_{5}(r)=\frac{\pi^{2}}{\ell_{\mathrm{P}}^{4} A^{\prime}(r)^{4}}\left(1-4 \hat{\lambda} L^{2} A^{\prime}(r)^{2}-3\left(3 \mu_{1}-12 \mu_{2}-4 \mu_{3}\right) L^{4} A^{\prime}(r)^{4}\right) .
$$

Let us examine the interactions that result in our toy model (3.1) after the unitarity constraints (3.11-3.16) are imposed. First as already noted above, the constraints (3.11) on the $\lambda_{i}$ couplings require that the curvature-squared interaction takes precisely the form of that appearing in Gauss-Bonnet gravity (2.7). Hence these interactions make two-derivative contributions to the equations of motion in a general background for $d \geq 4$ [22]. As also discussed above, for $d=3$, this term is a topological invariant in the four-dimensional bulk and so does not contribute to the equations of motion. However, it still plays a role in fixing our normalization for $a_{3}(r)$, as given in eq. 3.27. Note that for $d=2$, this interaction simply vanishes because of a Schouten identity for curvatures in three or fewer dimensions. One can understand this heuristically as arising because if one attempts to evaluate

$$
\varepsilon^{a_{1} a_{2} a_{3} a_{4}} \varepsilon_{b_{1} b_{2} b_{3} b_{4}} R_{a_{1} a_{2}}^{b_{1} b_{2}} R_{a_{3} a_{4}}^{b_{3} b_{4}}
$$

${ }^{8}$ The full story must be more involved since it seems that with a large coefficient, this term would lead to violations of the second law in black hole mergers. Hence it seems that if this topological term were to appear in the action of a complete theory of quantum gravity, the corresponding dimensionless coupling must be restricted to be relatively small. 
in less than four dimensions, the result must vanish because the indices do not run over enough values.

Turning to curvature-cubed interactions where the eight $\mu_{i}$ couplings are constrained by the five equations (3.12-3.16). Hence in general, one expects a threeparameter family of unitary $R^{3}$ interactions, which we will describe in terms of a basis of three independent interactions. As the first of these, one can readily verify that the cubic Lovelock interaction satisfies these constraints. This interaction is proportional to the six-dimensional Euler density $\mathcal{X}_{6}$ with

$$
\mu_{1}=-8, \mu_{2}=4, \mu_{3}=-24, \mu_{4}=3, \mu_{5}=24, \mu_{6}=16, \mu_{7}=-12, \mu_{8}=1 .
$$

In analogy to the comments about the Gauss-Bonnet interactions, this particular curvature-cubed interaction will only contribute two-derivative terms to the equations of motion in a general background for $d \geq 6[22]$. For $d=5$, it does not contribute to the equations of motion but still plays a role in determining $a_{5}(r)$, as given in eq. 3.28. For $d \leq 4$, this term simply vanishes and so should not be counted as one of the basis interactions.

As a second basis interaction, we can take the quasi-topological term $\mathcal{Z}_{d+1}$ given in eq. (2.8), which also satisfies the constraints (3.12-3.16). While this term only makes two derivative contributions to the gravitational equations in any RG flow geometry, we should recall that fourth order terms can appear in other backgrounds [15, 16]. Further, we should note that this term was only constructed for $d=4$ and $d \geq 6$ and so it cannot be counted amongst the basis interactions in $d \leq 3$ or $d=5$.

There are, in fact, two other candidates for the third basis interaction both of which are constructed from Weyl tensor: ${ }^{9}$

$$
\mathcal{W}_{1}=W_{a b}^{c d} W_{c d}^{e f} W_{e f}^{a b}, \quad \mathcal{W}_{2}=W_{a b}^{c d} W_{c d}^{e f} W_{e f}^{a b}
$$

In fact, these terms do not contribute to the linearized equations of motion around the RG flow geometry (2.2) at all, which can be deduced as follows. These backgrounds are conformally flat and since these terms are cubic in the Weyl tensor, the contribution to the quadratic action (3.25) for the graviton fluctuations must be proportional to at least one power of the Weyl tensor. Therefore there can be no contribution to the linearized equations of motion. Similarly, these terms do not contribute to the background equations which determine $A(r)$ for a particular RG flow and so the corresponding coupling

\footnotetext{
${ }^{9}$ Recall the definition of Weyl tensor in a $(d+1)$-dimensional spacetime is

$$
W_{a b c d}=R_{a b c d}-\frac{2}{d-1}\left(g_{a[c} R_{d] b}-g_{b[c} R_{d] a}\right)+\frac{2}{d(d-1)} R g_{a[c} g_{d] b}
$$

using the standard notation: $X_{[a b]}=\frac{1}{2}\left(X_{a b}-X_{b a}\right)$.
} 
constant would not appear in $\hat{\mu}$ in determining the curvature scale of the fixed points (3.4) or in the flow function (3.17). However, we emphasize that these interactions (3.32) would effect other properties of the boundary QFT. For example, they would contribute in a calculation of the three-point function of the stress tensor.

Now it may seem that we have an overabundance of basis interactions, since we have enumerated four possible unitary interactions above but our initial count of the constraints indicated that there should only be a three parameter family of such interactions. However, as noted in [23], the interactions listed above are not all independent for $d \geq 6$. In particular, in this case, we have the relation

$$
\mathcal{Z}_{d+1}=\mathcal{W}_{1}+\frac{3 d^{2}-9 d+4}{8(2 d-1)(d-3)(d-4)}\left(\mathcal{X}_{6}+8 \mathcal{W}_{1}-4 \mathcal{W}_{2}\right)
$$

Hence we can use any three of the above interactions as our basis for the curvaturecubed interactions in our holographic model with unitary RG flows when $d \geq 6$. For $d=5, Z_{6}$ is not defined and so our basis would be $\mathcal{X}_{6}, \mathcal{W}_{1}$ and $\mathcal{W}_{2}$. For $d<5$ Schouten identities reduce the number of number of possible interactions with $\mathcal{X}_{6}=0$ and $\mathcal{W}_{1}=\mathcal{W}_{2}$ [30]. Hence for $d=4$, we would have a two parameter family of interactions with $\mathcal{Z}_{5}$ and $\mathcal{W}_{1}$. For $d=3, \mathcal{Z}_{4}$ is also not defined and so we are reduced to a one parameter family with only $\mathcal{W}_{1}[30]$.

To close this section, we re-iterate that having found that $a_{d}^{*}$ satisfies a c-theorem (3.21) for our generalized holographic models, one must again ask what this quantity corresponds to in the boundary CFT. Motivated by Cardy's conjecture (1.5) and our results in the previous section, we first compare $a_{d}^{*}$ to the central charge $A$ for even $d$. With the approach of [29], we again find a precise match

$$
a_{d}^{*}=A \quad \text { for even } d
$$

Hence again, we have found evidence to support Cardy's conjecture with this broad class of holographic CFT's. However, we are again left without an interpretation of $a_{d}^{*}$ for odd $d$.

\section{4. $a_{d}^{*}$ and Entanglement Entropy - Take One}

Above, we have identified a quantity $a_{d}^{*}$ in eq. (3.20) for a broad class of holographic models which varies monotonically in RG flows. For even $d$, we have shown this quantity equals the coefficient of the A-type trace anomaly. However, a broader definition of $a_{d}^{*}$ is required to interpret our results when the boundary theory has a odd number of spacetime dimensions. We address this question here and in the next section, where 
we show that $a_{d}^{*}$ emerges in a certain calculation of entanglement entropy. Note that our discussion here and in the next section does not make use of the usual holographic calculation of entanglement entropy $[17,18]$. The latter only applies for Einstein gravity while here our bulk theory involves higher curvature interactions. Of course, if we eliminate these additional terms, our results for the entanglement entropy reduce to those calculated with the standard approach [17, 18].

We begin with the following observation: Recall quasi-topological gravity theory (2.6) for which we considered holographic c-theorems in section 2. Black hole solutions and thermodynamics were studied in some detail for this theory in [15]. The horizon entropy for any of the (static) black hole solutions found there is given by the following:

$$
S=\frac{2 \pi}{\ell_{\mathrm{P}}^{d-1}}\left(1+2 \frac{d-1}{d-3} \lambda k \frac{L^{2}}{r_{h}^{2}}-\frac{3(d-1)}{d-5} \mu k^{2} \frac{L^{4}}{r_{h}^{4}}\right) \oint d^{d-1} x \sqrt{h\left(r_{h}\right)} .
$$

where $r_{h}$ is the horizon radius. The final factor yields the 'area' of the horizon with $h_{a b}\left(r_{h}\right)$ being the induced metric on a spatial slice of the horizon. Also $k$ is an integer with values $+1,0$ or -1 for the horizon geometry being spherical (i.e., $S^{d-1}$ ), planar (i.e., $R^{d-1}$ ) or hyperbolic (i.e., $H^{d-1}$ ), respectively. From this result (4.1), we see that with a hyperbolic horizon if we set the horizon radius to match the AdS curvature scale, i.e., $r_{h}=\tilde{L}=L / f_{\infty}^{1 / 2}$, then the factor in brackets above becomes $1-\frac{2(d-1)}{d-3} \lambda f_{\infty}-$ $\frac{3(d-1)}{d-5} \mu f_{\infty}^{2}$. From eq. (2.14), we recognize that this is precisely the same factor appearing in the fixed point value of our flow function. Hence, with these choices, we find that the entropy density can be written as:

$$
\begin{aligned}
S & =\frac{2 \pi}{\pi^{d / 2}} \frac{\Gamma(d / 2)}{\tilde{L}^{d-1}} a_{d}^{*} \int d^{d-1} x \sqrt{h\left(r_{h}\right)} \\
& =(4 \pi)^{d / 2} \Gamma(d / 2) T^{d-1} a_{d}^{*} \int d^{d-1} x \sqrt{h\left(r_{h}\right)}
\end{aligned}
$$

where $T=1 /(2 \pi \tilde{L})$ is the Hawking temperature of the horizon - the latter is fixed by the choice $r_{h}=\tilde{L}$. The interpretation in terms of the dual CFT is that eq. (4.2) gives the entropy of a thermal bath at temperature $T$ in a background geometry $R \times H^{d-1}$, where, as we will see, the curvature scale of the hyperbolic geometry is precisely $1 / \tilde{L}$.

Note that the choice of horizon radius above also fixes the mass parameter in the black hole metric to be zero. That is, the solution actually reduces to a hyperbolic foliation of pure $\mathrm{AdS}_{d+1}$, i.e.,

$$
d s^{2}=\frac{d r^{2}}{\left(\frac{r^{2}}{\tilde{L}^{2}}-1\right)}-\left(\frac{r^{2}}{\tilde{L}^{2}}-1\right) d t^{2}+r^{2} d \Sigma_{d-1}^{2}
$$




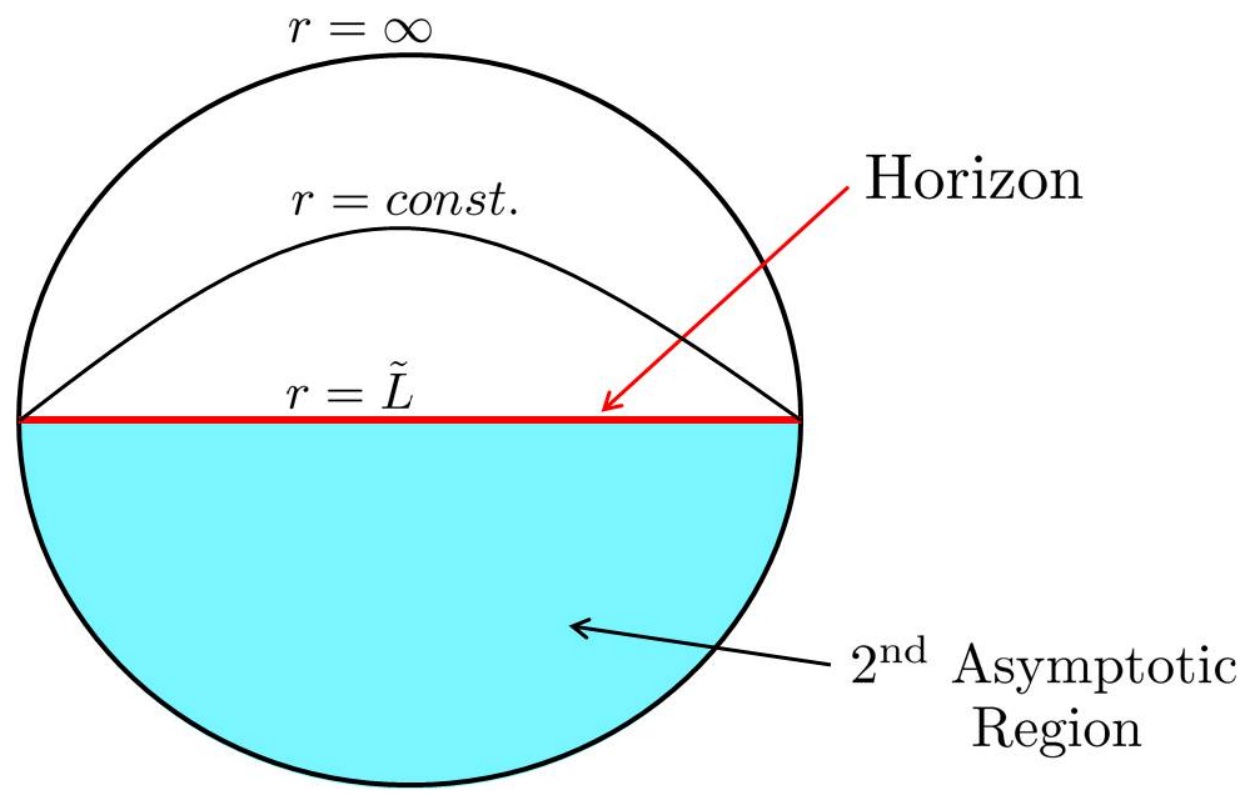

Figure 1: A slice of constant $t$ through the $\operatorname{AdS}_{d+1}$ metric in eq. (4.3).

where $d \Sigma_{d-1}^{2}$ denotes the line element on $H^{d-1}$ with unit curvature. There is a puzzle here as we have just said the bulk geometry corresponds to the AdS vacuum and so we would expect that the boundary theory is also in its vacuum state. From this perspective, one must ask why should there be a nonvanishing entropy density at all? The answer to the latter question can be found in considering the constant time slice of eq. (4.3) shown in figure 1. This constant $t$ slice is similar to the Einstein-Rosen bridge in a Schwarzschild black hole [19]. The (spatial section of the) full AdS boundary has the topology $S^{d-1}$. However, as illustrated in the figure, with the hyperbolic foliation (4.3), only half of this boundary is reached in the limit $r \rightarrow \infty$. The other half is reached from the second asymptotic region 'behind the horizon' at $r_{h}=\tilde{L}$. Hence a natural interpretation of the entropy is that it corresponds to the entanglement entropy ${ }^{10}$ of the CFT (in its vacuum state) between these two halves of the $S^{d-1}$.

Of course, to evaluate the entropy in eq. (4.2), we must perform the integral over the horizon. It is no surprise that the latter yields an infinite result since, as illustrated in figure 1, the hyperbolic horizon extends out to the AdS boundary. Let us write the induced metric on the bifurcation surface of the event horizon as

$$
r_{h}^{2} d \Sigma_{d-1}^{2}=\tilde{L}^{2}\left(\frac{d \rho^{2}}{1+\rho^{2}}+\rho^{2} d \Omega_{d-2}^{2}\right)
$$

\footnotetext{
${ }^{10}$ Related ideas were discussed in [37, 38].
} 
where $d \Omega_{d-2}^{2}$ denotes the line element on a unit $(d-2)$-sphere. ${ }^{11}$ Integrating over the horizon out to some maximum radius $\rho_{\max }$ yields

$$
S=\frac{2 \pi}{\pi^{d / 2}} \Gamma(d / 2) a_{d}^{*} \Omega_{d-2} \int_{0}^{\rho_{\max }} \frac{\rho^{d-2} d \rho}{\sqrt{1+\rho^{2}}},
$$

where $\Omega_{d-2}=2 \pi^{(d-1) / 2} / \Gamma((d-1) / 2)$ is the area of a unit $(d-2)$-sphere.

As already noted, the bifurcation surface of the hyperbolic horizon is a surface that stretches across the entire AdS geometry and, so in particular, extends out to the boundary. Hence we can interprete the maximum radius $\rho_{\max }$ above in terms of a UV cut-off in the boundary theory. Using the standard UV/IR conversion, we have a short-distance cut-off: $\delta=\tilde{L} / \rho_{\max }$. Then we observe that the leading contribution arising from eq. (4.5) can be written as

$$
S \simeq \frac{2 \pi}{\pi^{d / 2}} \frac{\Gamma(d / 2)}{d-2} a_{d}^{*} \frac{\mathcal{A}_{d-2}}{\delta^{d-2}}+\cdots
$$

where $\mathcal{A}_{d-2}=\Omega_{d-2} \tilde{L}^{d-2}$ is the 'area' of the equator dividing the two halves of the $S^{d-1}$ in the boundary theory. This leading divergence takes precisely the form expected for the 'area law' contribution to the entanglement entropy in a $d$-dimensional CFT [18, 39]. Note that the hyperbolic geometry of the horizon was essential to ensure the leading power was $1 / \delta^{d-2}$ here despite the area integral being $(d-1)$-dimensional in eq. (4.5). This divergent contribution to the entanglement entropy is not universal - e.g., see $[18,39]$. However, a universal contribution can be extracted from the subleading terms. The form of the universal contribution to the entanglement entropy depends on whether $d$ is odd or even $[18,39]$ :

$$
S_{\text {univ }}= \begin{cases}(-)^{\frac{d}{2}-1} 4 a_{d}^{*} \log (2 \tilde{L} / \delta) & \text { for even } d, \\ (-)^{\frac{d-1}{2}} 2 \pi a_{d}^{*} & \text { for odd } d .\end{cases}
$$

Up to this point the discussion focussed on quasi-topological gravity theory (2.6), for which a broad class of black hole solutions is known [15]. However, at the end of the day, our analysis of the entanglement entropy only makes reference to the $\mathrm{AdS}_{d+1}$ vacuum solution. This suggests that our result should extend to a wider class of gravitational theories, as we will now show in the following.

In particular, consider any of the theories considered in section 3 and present the $\mathrm{AdS}_{d+1}$ vacuum in the hyperbolic foliation of eq. (4.3). Treating the latter as a black

\footnotetext{
${ }^{11}$ It is useful to note that this spatial metric on the horizon precisely matches with the spatial metric in the boundary theory.
} 
hole, the horizon entropy can be calculated using Wald's entropy formula [35]

$$
S=-2 \pi \int_{\text {horizon }} d^{d-1} x \sqrt{h} \frac{\partial \mathcal{L}}{\partial R^{a b}{ }_{c d}} \hat{\varepsilon}^{a b} \hat{\varepsilon}_{c d},
$$

which applies very generally for any (covariant) theory of gravity - this was one of two approaches considered in [15] to derive eq. (4.1). Note that above, $\mathcal{L}$ denotes the gravitational Lagrangian and $\hat{\varepsilon}_{a b}$ is the binormal to the horizon. Of course, with the given metric (4.3), the integrand in eq. (4.8) is constant across the horizon and so the total entropy diverges as described above. However, we use this this constancy to rewrite eq. (4.8) in a way that facilitates comparisons with eq. (4.2)

$$
S=-\left.2 \pi \frac{\partial \mathcal{L}}{\partial R_{c d}^{a b}} \hat{\varepsilon}^{a b} \hat{\varepsilon}_{c d} \int d^{d-1} x \sqrt{h}\right|_{\text {horizon }},
$$

Now in section 3, we observed in eq. (3.26) that at any point in the RG flow geometries that the pre-factor appearing in the above expression. To be precise, one finds

$$
\frac{\partial \mathcal{L}}{\partial R^{t r}{ }_{t r}}=2 \frac{\Gamma(d / 2)}{\pi^{d / 2}} A^{\prime}(r)^{d-1} a_{d}(r) .
$$

Hence if we consider an $\mathrm{AdS}_{d+1}$ solution dual to one of the RG fixed points, the expression above can be considered at the hyperbolic horizon and the pre-factor in eq. (4.9) becomes precisely that appearing in eq. (4.2). Hence all of previous discussion, including the precise numerical factors, extends to any of the theories in section 3 . In particular then, we have the same identification of $a_{d}^{*}$ with a universal coefficient in the entanglement entropy for these theories as well.

To recap our result here: We interpret the horizon entropy of the hyperbolic foliation of $\mathrm{AdS}_{d+1}$ as the entanglement entropy of the boundary CFT (in its vacuum state) between the two halves of the $S^{d-1}$. With this framework, we are then able to identify the coefficient $a_{d}^{*}$ in terms of universal contribution to the entanglement entropy (4.7). In particular though, this identification can be applied for both odd and even $d$. Further, while the analysis was originally made for quasi-topological gravity, it extends to any of the gravitational theories considered in section 3.

\section{5. $a_{d}^{*}$ and Entanglement Entropy - Take Two}

In the previous section, we have argued that $a_{d}^{*}$, the quantity which appeared in the holographic c-theorem in sections 2 and 3, can be identified with a coefficient in a particular calculation of entanglement entropy in the dual CFT. The derivation there relied on relating the entanglement entropy for the vacuum in $R \times S^{d-1}$ to the thermal 


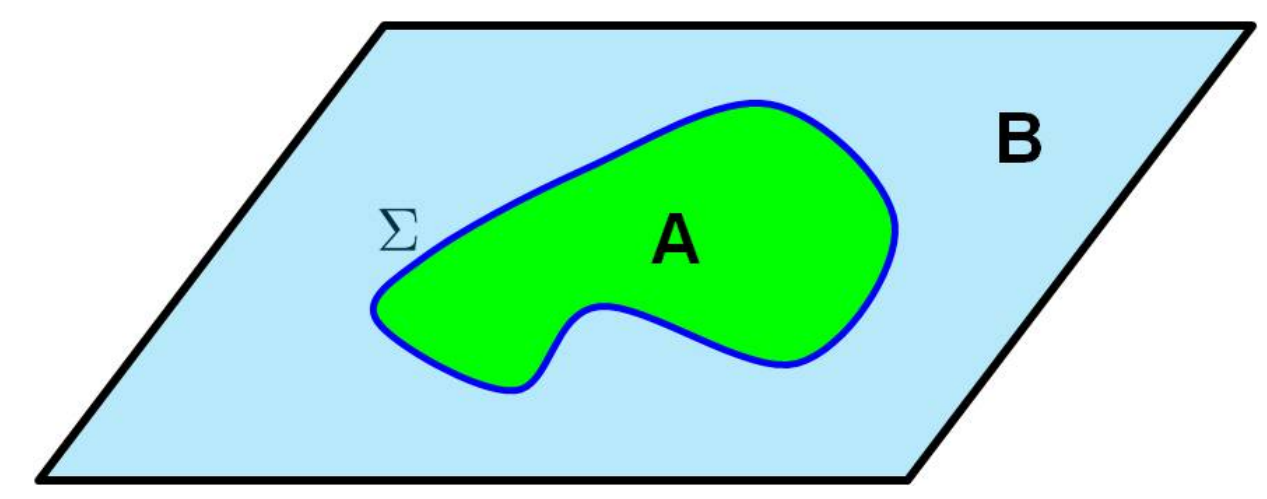

Figure 2: The division of a particular time slice into two regions $A$ and $B$.

entropy of a heat bath in $R \times H^{d-1}$. While this discussion can be made more precise [40], we would like to frame the same identification within a more conventional calculation of entanglement entropy in this section.

Generally entanglement entropy arises as follows [41, 39]: One divides a given system into two parts, say, A and B, and integrates out the degrees of freedom in one subsystem, B. The remaining degrees of freedom in A are described by a density matrix $\rho_{\mathrm{A}}$. The entanglement entropy is then simply the von Neumann entropy of this density matrix, i.e., $S=-\operatorname{Tr}\left[\rho_{\mathrm{A}} \log \rho_{\mathrm{A}}\right]$. In field theories, the system is typically subdivided by introducing a boundary $\Sigma$ which separates the space (i.e., a constant time slice) into two regions, as shown in figure 2.

A standard approach to calculating the entanglement entropy is to apply the replica trick [41]. Since the operator $\log \rho_{\mathrm{A}}$ often lacks a clear definition, this construction begins by considering (integer) powers of the density matrix, which may be defined as

$$
\operatorname{Tr}\left[\rho_{\mathrm{A}}^{n}\right]=Z_{n} /\left(Z_{1}\right)^{n}
$$

Here $Z_{n}$ is the partition function of $n$ copies of the equivalent system satisfying the constraint that their degrees of freedom are identified on the region B. That is, one calculates the partition function $Z_{n}$ on an $n$-fold cover of the Euclidean ${ }^{12}$ background geometry where a cut is introduced throughout region $\mathrm{B}$ at $t_{\mathrm{E}}=0$. At the cut, the copy $n$ of the fields is connected to copy $n+1$ when approaching from $t_{\mathrm{E}} \rightarrow 0^{-}$and to copy $n-1$ when approaching from $t_{\mathrm{E}} \rightarrow 0^{+}$. The factors of $Z_{1}$ appear in the denominator of eq. (5.1) to ensure that the density matrix is properly normalized with $\operatorname{Tr}\left[\rho_{\mathrm{A}}\right]=1$. Assuming that the expressions calculated in eq. (5.1) can be extended to real $n$, the

\footnotetext{
${ }^{12}$ An implicit initial step in this calculation was to Wick rotate the time coordinate to $t_{\mathrm{E}}=i t$.
} 
entanglement entropy can then be calculated as

$$
S=-\lim _{n \rightarrow 1} \frac{\partial}{\partial n} \operatorname{Tr}\left[\rho_{\mathrm{A}}^{n}\right]=-\lim _{n \rightarrow 1}\left(\frac{\partial}{\partial n}-1\right) \log Z_{n} .
$$

Hence the calculation of the entanglement entropy can be summarized with the following schematic outline:

1. Construct the $n$-fold cover described above.

2. Calculate the partition function $Z_{n}$ on this $n$-fold cover.

3. Analytically continue $Z_{n}$ to real $n$.

4. Evaluate the entanglement entropy using eq. (5.2).

While the above approach is customary in the condensed matter literature [41], a common modification is prevalent in the high energy physics literature which gives these calculations a geometric character [42]. Hence, we denote this the 'geometric approach' in the following. Essentially one interchanges the steps 2 and 3 above and proceeds with the following calculation:

1. Construct the $n$-fold cover described above.

$2^{\prime}$. Analytically continue this background geometry to real $n$.

$3^{\prime}$. Calculate the partition function $Z_{n}$ on the analytically continued cover.

4. Evaluate the entanglement entropy using eq. (5.2).

From this geometric perspective, the boundary $\Sigma$ is codimension two surface where the $n$-fold cover introduces an angular excess of $2 \pi(n-1)$. The analytic continuation in step $2^{\prime}$ assumes that we can define the corresponding geometry with an arbitrary angular (or deficit) excess at this surface. Given the limit $n \rightarrow 1$ in eq. (5.2), we need only consider values of $n$ where $n=1-\epsilon$ with $\epsilon \ll 1$. Hence we can think that the entanglement entropy measures the response of the field theory to an infinitesimal conical defect at $\Sigma$.

However, we must emphasize that we are only fully justified in using this revised calculation if there is a rotational symmetry around the surface $\Sigma$. Without such a symmetry, the analytic continuation of the geometry in step $2^{\prime}$ is not well defined.

The preceding discussion is general and so now we turn to the case of interest which appeared in section 4 . There we have the $d$-dimensional boundary CFT on $R \times S^{d-1}$. 
As above, we Wick rotate to Euclidean time, $t_{\mathrm{E}}=i t$, so that the background metric becomes

$$
d s^{2}=d t_{\mathrm{E}}^{2}+\tilde{L}^{2} d \Omega_{d-1}^{2} .
$$

At $t_{\mathrm{E}}=0$, we divide the sphere into two equal halves along the equator of $S^{d-1}$, which is then the surface $\Sigma$ in the nomenclature introduced above. Now we would like to calculate the entanglement entropy with the geometric approach. However, our background geometry does not have the desired rotational symmetry around $\Sigma$ and so as it stands this calculation would be ill-defined. Now, we note that the field theory of interest is a CFT and so it is possible to use the conformal symmetry to transform to a geometry with the desired symmetry. In particular, we make the coordinate transformation

$$
t_{\mathrm{E}}=\tilde{L} \log \left(\frac{1-\cos \theta}{\sin \theta}\right)
$$

with which the metric (5.3) becomes

$$
d s^{2}=\frac{d \tilde{s}^{2}}{\sin ^{2} \theta} \quad \text { with } \quad d \tilde{s}^{2}=\tilde{L}^{2}\left(d \theta^{2}+\sin ^{2} \theta d \Omega_{d-1}^{2}\right) .
$$

Hence with a conformal transformation, the background geometry has become a $d$ dimensional sphere of radius $\tilde{L}$. Note that with the transformation (5.4), the surface $t_{\mathrm{E}}=0$ corresponds to $\theta=\pi / 2$ and the Weyl factor in eq. (5.5) is simply one there, i.e., the conformal transformation leaves the geometry on the $t_{\mathrm{E}}=0$ surface unchanged. Further $t_{\mathrm{E}} \rightarrow \pm \infty$ corresponds to $\theta=0$ and $\pi$. That is, the spherical boundaries at $t_{\mathrm{E}} \rightarrow$ $\pm \infty$ in the original background geometry have been compactified to the points at the poles of the $d$-sphere. Implicitly, our compactification also inserts the identity operator at the poles so that there is nothing exceptional about these two positions in the smooth $d$-sphere geometry. These insertions can be viewed from another perspective: Implicit in our discussion here is that the boundary field theory is in its vacuum state. From this point of view, we can ensure that the CFT is in its vacuum state on the $t_{\mathrm{E}}=0$ surface by imposing vacuum boundary conditions at $\left|t_{\mathrm{E}}\right| \rightarrow \infty$ in the $R \times S^{d-1}$ background. Upon compactifying the geometry to $S^{d}$, inserting identity operators at the poles simply conforms to this choice of vacuum boundary conditions.

Of course, the $d$-sphere geometry has the desired rotational symmetry and so we are free to consider the geometric approach for calculating the entanglement entropy. At this point, we have set the stage to evaluate the entanglement entropy purely from the perspective of any conformal field theory (without referring to holography) and we turn to a non-holographic calculation in section 5.4. Here we continue towards realizing this calculation in the framework of the AdS/CFT correspondence. However, after describing the holographic calculation, we will make a brief diversion to collect a 
number of apparently unrelated results. With these, we can easily compare the results for the entanglement entropy found here to those in the previous section.

At the outset, let us generalize the discussions. In particular, in the following we will allow the bulk gravitational theory to be described by any general covariant action:

$$
I=\int d^{d+1} x \sqrt{-g} \mathcal{L}\left(g^{a b}, R_{c d}^{a b}, \nabla_{e} R_{c d}^{a b}, \ldots, \text { matter }\right)+\text { boundary terms }
$$

For the purposes of comparison, we must remind the reader that the discussion of section 4 entailed a Minkowski signature both in the bulk and the boundary theory, as in eq. (5.6). However, for the above calculation in the boundary theory, we have Wick rotated to a Euclidean signature and it follows that the dual gravity calculations will also be in Euclidean signature. Hence we wish to distinguish the gravity action after Wick rotation as

$$
I_{\mathrm{E}}=\int d^{d+1} x \sqrt{g} \mathcal{L}_{\mathrm{E}}\left(g^{a b}, R_{c d}^{a b}, \nabla_{e} R_{c d}^{a b}, \ldots, \text { matter }\right)+\text { boundary terms } .
$$

We do so because there is a delicate issue of signs. Essentially, the Wick rotation introduces an extra minus sign in the action so that we have $\mathcal{L}_{\mathrm{E}}=-\mathcal{L}$. In other words, if one evaluates the Lagrangian density on the $\mathrm{AdS}_{d+1}$ vacuum (as we will do later on), then one finds $\mathcal{L}<0$ in Minkowski signature while $\mathcal{L}_{\mathrm{E}}>0$ in Euclidean signature.

Now within the AdS/CFT framework, the calculation of the entanglement entropy described for the boundary CFT above is translated to a gravitational calculation. We begin by considering the $\mathrm{AdS}_{d+1}$ vacuum in Euclidean signature with metric

$$
d s^{2}=\frac{d r^{2}}{\left(\frac{r^{2}}{\tilde{L}^{2}}+1\right)}+r^{2} d \Omega_{d}^{2} .
$$

Here $d \Omega_{d}^{2}$ denotes the line element on a $d$-sphere with unit curvature. This foliation was chosen as it is naturally dual to the boundary CFT on an $S^{d}$ background geometry (of radius $\tilde{L}$ ), as desired above. As usual [43], the path integral over the bulk gravity fields is treated in the saddle-point approximation and hence we have

$$
Z_{n}=\exp \left[-I_{\mathrm{E}, n}\right]
$$

where $I_{\mathrm{E}, n}$ is the Euclidean action (5.7) evaluated on-shell with the $n$-fold cover of the $\mathrm{AdS}_{d+1}$ space, where as above $n$ is real. In the previous construction with the boundary CFT, the surface $\Sigma$ was a maximal $S^{d-1}$ in the $d$-sphere. Constructing the $n$-fold cover then introduces a conical singularity at $\Sigma$ with an angular excess of $2 \pi(n-1)$. Here our holographic dual extends this conical singularity to a bulk surface $\Sigma_{b}$ covers the same 
maximal $S^{d-1}$ (in the spherical portion of the foliation (5.8)) and the radial coordinate in the $\mathrm{AdS}_{d+1}$ geometry. ${ }^{13}$ However, without a full understanding of string theory or quantum gravity in the AdS bulk, we will not know how to resolve the conical curvature singularity produced at finite $n$ and so it is not really possible to define the saddle-point action in eq. (5.9). Fortunately, as described above, to calculate the entanglement entropy using the geometric approach, we really only need to measure the response of the system to an infinitesimal conical defect at $\Sigma$ and $\Sigma_{b}$ (i.e., with $n=1-\epsilon$ with $\epsilon \ll 1$ ). As we describe next, such an infinitesimal angular deficit can be treated in a well-defined way following the approach of [45].

\subsection{Conical defect in $\operatorname{AdS}_{d+1}$}

Motivated by calculations of black hole entropy, ref. [45] developed a description of Riemannian geometry in the presence of a conical defect. Their prescription involved replacing the singular geometry with a 'regulator' geometry, which smoothed out the region around the conical singularity, and then carefully considering the limit in which this smooth geometry converges towards the original singular manifold. This allows one to evaluate certain integrals of covariant functionals constructed from the background geometry even in the presence of the conical defect.

To establish some notation, let us denote the full geometry as $\mathcal{M}$ and the singular surface as $\Sigma_{b}$, to be consistent with the holographic discussion above. As above, we assume there is a rotational symmetry about the singularity and we set the angle around $\Sigma_{b}$ to run through a range $2 \pi(1-\epsilon)$. Now the discussion in [45] considered conical defects where $\epsilon$ was small but finite. It was found that in a general integral, such as $\int_{\mathcal{M}} R^{2}$, the contribution linear in $\epsilon$ is independent of the regulator but higher order terms depend on the details of the smoothing geometry. That is, their construction demonstrates that in such integrations, the only universally defined result is that where conical singularity makes a linear contribution. With this proviso, a key result of [45] is that the curvature of $\mathcal{M}$ can be represented as

$$
{ }^{(\epsilon)} R_{c d}^{a b}=R^{a b}{ }_{c d}+2 \pi \epsilon \sum_{i, j=1}^{2}\left(\left[n^{i}\right]^{a}\left[n^{i}\right]_{c}\left[n^{j}\right]^{b}\left[n^{j}\right]_{d}-\left[n^{i}\right]^{a}\left[n^{i}\right]_{d}\left[n^{j}\right]^{b}\left[n^{j}\right]_{c}\right) \delta_{\Sigma_{b}}
$$

where $R^{a b}{ }_{c d}$ is the smooth contribution away from $\Sigma_{b}$ and $\delta_{\Sigma_{b}}$ is a two-dimensional $\delta$-function satisfying $\int_{\mathcal{M}} f(x) \delta_{\Sigma_{b}}=\int_{\Sigma_{b}} f(x)$. The $\left[n^{i}\right]_{a}$ with $i=1,2$ are a pair of orthogonal unit vectors (i.e., $\left[n^{i}\right]_{a}\left[n^{j}\right]^{a}=\delta^{i j}$ ) spanning the space transverse to $\Sigma_{b}$. Again,

\footnotetext{
${ }^{13}$ Note that one does not necessarily have to introduce a bulk singularity in these calculations of entanglement entropy, as illustrated in [44].
} 
note that this formula (5.10) must be applied with some care. Certainly expressions where higher powers of $\delta_{\Sigma_{b}}$ appear will not be well-defined. ${ }^{14}$

Now a short calculation allows us re-express eq. (5.10) as follows

$$
{ }^{(\epsilon)} R^{a b}{ }_{c d}=R^{a b}{ }_{c d}+2 \pi \epsilon \tilde{\varepsilon}^{a b} \tilde{\varepsilon}_{c d} \delta_{\Sigma_{b}}
$$

where $\tilde{\varepsilon}_{a b} \equiv\left[n^{1}\right]_{a}\left[n^{2}\right]_{b}-\left[n^{2}\right]_{a}\left[n^{1}\right]_{b}$ is the two-dimensional volume form in the (Euclidean) space transverse to $\Sigma_{b}$. This form will provide a more convenient expression for the entanglement entropy below.

Returning to our holographic calculation, we wish to evaluate the gravitational action for $\mathrm{AdS}_{d+1}$ space with a conical singularity. As illustrated in [45], the issue of higher powers of $\delta_{\Sigma_{b}}$ can be avoided with a carefully engineered action, such as in Lovelock gravity [22] where the Lagrangian has a topological origin. However, this problem certainly arises for the general covariant action (5.7) which we are considering here. Fortunately, we do not need to consider finite $\epsilon$ rather we seek precisely the linear response of the system to the introduction of the conical singularity. Combining eqs. (5.2) and (5.9), the holographic calculation of entanglement entropy requires that we evaluate

$$
S=-\lim _{\epsilon \rightarrow 0}\left(\frac{\partial}{\partial \epsilon}+1\right) I_{\mathrm{E}, 1-\epsilon} .
$$

With the limit $\epsilon \rightarrow 0$, the (single) derivative picks out only the linear contribution of the conical singularity in the action.

Given eq. (5.11) above, it is straightforward to show that

$$
\begin{aligned}
I_{\mathrm{E}, 1-\epsilon} & =(1-\epsilon) I_{\mathrm{E}, 1}+\int d^{d+1} x \sqrt{g} \frac{\partial \mathcal{L}_{\mathrm{E}}}{\partial R^{a b} c d} 2 \pi \epsilon \tilde{\varepsilon}^{a b} \tilde{\varepsilon}_{c d} \delta_{\Sigma_{b}}+O\left(\epsilon^{2}\right) \\
& =(1-\epsilon) I_{\mathrm{E}, 1}+2 \pi \epsilon \int_{\Sigma_{b}} d^{d-1} x \sqrt{h} \frac{\partial \mathcal{L}_{\mathrm{E}}}{\partial R^{a b}{ }_{c d}} \tilde{\varepsilon}^{a b} \tilde{\varepsilon}_{c d}+O\left(\epsilon^{2}\right) .
\end{aligned}
$$

Above there are two sources of $O(\epsilon)$ contributions to $I_{\mathrm{E}, 1-\epsilon}$. First, the angle around $\Sigma_{b}$ runs through the range $2 \pi(1-\epsilon)$ and since we have a rotational symmetry in this direction, the smooth contributions to the curvature in eq. (5.11) yield the term $(1-\epsilon) I_{\mathrm{E}, 1}$. Then there is the term linear in $\delta_{\Sigma}$ where each appearance of the curvature in the Lagrangian is replaced in turn by the $\delta$-function term on the right-hand side of eq. (5.11). The latter is yields the differentiation of the Lagrangian with respect to $R_{c d}^{a b}$.

\footnotetext{
${ }^{14}$ It was later realized that this result also only applies in situations where the extrinsic curvature of $\Sigma_{b}$ vanishes [46].
} 
Now substituting the above result (5.13) into eq. (5.12), our AdS/CFT calculation of the entanglement entropy yields

$$
S=-\left.2 \pi \frac{\partial \mathcal{L}_{\mathrm{E}}}{\partial R^{a b}{ }_{c d}} \tilde{\varepsilon}^{a b} \tilde{\varepsilon}_{c d} \int d^{d-1} x \sqrt{h}\right|_{\Sigma_{b}} .
$$

This expression is evaluated in the $\mathrm{AdS}_{d+1}$ vacuum solution (5.8) (with $\epsilon=0$ ). Hence the pre-factor above is constant and so we have pulled it out of the integral over $\Sigma_{b}$.

We have expressed this result (5.14) in a way that it appears to have the same form as the formula for the Wald entropy in eq. (4.9). Of course, the present discussion made no reference to a black hole geometry or black hole thermodynamics. We must also emphasize that there are subtle differences arising because the spacetime signature differs between the two expressions. In particular then, eq. (4.9) contains the variation of the Minkowski-signature Lagarangian $\mathcal{L}$ while eq. (4.9) contains that for Euclideansignature Lagarangian $\mathcal{L}_{\mathrm{E}}$. As described above, there is a difference of sign between these two, i.e., $\mathcal{L}_{\mathrm{E}}=-\mathcal{L}$. However, this minus sign is compensated by an extra sign that arises in Wick rotating the two transverse volume forms appearing in each expression e.g., in Minkowski signature, we have $\hat{\varepsilon}^{a b} \hat{\varepsilon}_{a b}=-2$ while $\tilde{\varepsilon}^{a b} \tilde{\varepsilon}_{a b}=2$ in Euclidean signature. Further, one can verify that upon analytically continuing the Euclidean $\operatorname{AdS}_{d+1}$ metric back to Minkowski signature, the surface $\Sigma_{b}$ above coincides with the bifurcation surface of the hyperbolic black hole considered in section 4 . Hence the expression in eq. (5.14) above and that in eq. (4.9) are evaluated on precisely the same geometry and yield precisely the same result for the entanglement entropy. ${ }^{15}$ We emphasize again that the results here and in the previous section apply for a completely general covariant gravity theory (5.6). We will need two more results to connect eq. (5.14) to $a_{d}^{*}$ in this general context.

\subsection{AdS equations of motion}

Next we consider the equations of motion arising from the general action (5.6). In particular, we will organize the field equations so as to separate the contributions coming from the variations of the (inverse) metric $g^{a b}$ and the curvature $R_{c d}^{a b}$. Hence we write the variation of the action (5.6) as:

$$
\delta I=\int d^{d+1} x \sqrt{-g}\left[-\frac{1}{2} \mathcal{L} g_{a b} \delta g^{a b}+\frac{\delta \mathcal{L}}{\delta g^{a b}} \delta g^{a b}+\frac{\delta \mathcal{L}}{\delta R^{a b}{ }_{c d}} \delta R^{a b}{ }_{c d}\right]
$$

\footnotetext{
${ }^{15}$ The same analysis can be applied in a discussion of black hole entropy and our results readily confirm the equivalence of Wald's Noether charge method [35] and the off-shell approach [47], which introduces a conical singularity in the Euclidean black hole geometry. The equivalence of these two techniques was previously discussed in [48].
} 
Now a few comments are in order here: First we stress that we are not treating the curvature $R_{c d}^{a b}$ as an independent field. We are still making the standard variations above with

$$
\begin{gathered}
\delta R_{c d}^{a b}=g^{b e}\left(\nabla_{c} \delta \Gamma_{e d}^{a}-\nabla_{d} \delta \Gamma_{e c}^{a}\right)+R_{e c d}^{a} \delta g^{b e} \\
\delta \Gamma_{e d}^{a}=\frac{1}{2} g^{a f}\left(\nabla_{e} \delta g_{f d}+\nabla_{d} \delta g_{f e}-\nabla_{f} \delta g_{e d}\right)
\end{gathered}
$$

and $\delta g_{a b}=-g_{a c} g_{b d} \delta g^{c d}$. Our goal in writing eq. (5.15) is simply to organize the various contributions in equations of motion and in particular we wish to isolate those coming from the variations $\delta R^{a b}{ }_{c d}$. In particular, the expression $\delta \mathcal{L} / \delta R^{a b}{ }_{c d}$ above in eq. (5.15) is precisely same as that appearing in Wald's entropy formula (4.8). Another comment is that the notation above hides the result of any necessary integration by parts in $\delta \mathcal{L} / \delta g^{a b}$ and $\delta \mathcal{L} / \delta R^{a b}{ }_{c d}$. Finally, let us observe that given this approach, $\delta \mathcal{L} / \delta g^{a b}$ collects contributions from two sources: variations of the connections (5.17) appearing in covariant derivatives of the curvature tensor appearing in the action and variations of explicit metrics and connections which may appear in interactions involving the matter fields. Now it is straightforward to write out the general gravity equations of motion as

$$
\frac{1}{2} \mathcal{L} g_{a b}=\frac{\delta \mathcal{L}}{\delta g^{a b}}+2 g_{(a \mid d} \nabla^{e} \nabla_{c} \frac{\delta \mathcal{L}}{\delta R^{b) e}{ }_{c d}}-R_{(a \mid c d}^{e} \frac{\delta \mathcal{L}}{\delta R^{b) e}{ }_{c d}}
$$

Next, we wish to consider evaluating the equations in an $\operatorname{AdS}_{d+1}$ background which produces a number of simplifications. In particular, this background is a maximally symmetric geometry. Further any background tensors constructed from the geometry and/or the matter fields must respect the same symmetries and so in particular, any covariant derivatives of such quantities will vanish in general, i.e., $\nabla_{a}[\cdots]=0$. Hence the second term on the right-hand side of eq. (5.18) must vanish in this background. Further, it is clear that all of the contributions in $\delta \mathcal{L} / \delta g^{a b}$ will also automatically vanish in the $\operatorname{AdS}_{d+1}$ background. Finally, turning to the third term on the right-hand side, we set

$$
R_{c d}^{a b}=-\frac{1}{\tilde{L}^{2}}\left(\delta_{c}^{a} \delta_{d}^{b}-\delta_{d}^{a} \delta_{c}^{b}\right)
$$

for the $\mathrm{AdS}_{d+1}$ background. Now combining these three simplifications, the equations of motion (5.18) reduce to

$$
\left.\frac{\delta \mathcal{L}}{\delta R^{\left(a \mid e_{c d}\right.}}\right|_{A d S} g_{b) c} \delta^{e}{ }_{d}=-\left.\frac{\tilde{L}^{2}}{4} \mathcal{L}\right|_{A d S} g_{a b} .
$$

Hence we see that, in the $\mathrm{AdS}_{d+1}$ background, the equations of motion balance two sets of nontrivial contributions. We stress that despite our assumption that the background 
geometry corresponds to $\mathrm{AdS}_{d+1}$, eq. (5.20) is still a nontrivial equation. In particular, it determines the curvature scale for the vacuum solution(s).

We make one further step at this point. As commented above, all of the background tensors must respect the symmetries of the $\mathrm{AdS}_{d+1}$ background. Therefore we must have $\delta \mathcal{L} / \delta R^{a b}{ }_{c d} \propto\left(\delta_{a}{ }^{c} \delta_{b}{ }^{d}-\delta_{a}{ }^{d} \delta_{b}{ }^{c}\right)$ and we can re-express eq. (5.20) as

$$
\left.\frac{\delta \mathcal{L}}{\delta R^{a b}{ }_{c d}}\right|_{A d S}=-\left.\frac{\tilde{L}^{2}}{4 d} \mathcal{L}\right|_{A d S}\left(\delta_{a}{ }^{c} \delta_{b}{ }^{d}-\delta_{a}{ }^{d} \delta_{b}{ }^{c}\right) .
$$

Of course, the signature of the bulk spacetime has no effect on the preceding analysis and hence the same result follows equally well from the Euclidean signature action (5.7). For completeness, we explicitly write out the corresponding expression:

$$
\left.\frac{\delta \mathcal{L}_{\mathrm{E}}}{\delta R^{a b}{ }_{c d}}\right|_{A d S}=-\left.\frac{\tilde{L}^{2}}{4 d} \mathcal{L}_{\mathrm{E}}\right|_{A d S}\left(\delta_{a}{ }^{c} \delta_{b}{ }^{d}-\delta_{a}{ }^{d} \delta_{b}{ }^{c}\right) .
$$

\subsection{A-type trace anomaly}

Given our results in eqs. (2.16) and (3.34), we anticipate that the result for the entanglement entropy should be proportional to the $A$ coefficient in the trace anomaly (1.4) for even $d$. Here we simply point out the short cut to calculating $A$ presented in [29]. Given a general action (5.6) for the bulk gravity theory, $A$ can be determined by simply evaluating value the Lagrangian in the $\mathrm{AdS}_{d+1}$ vacuum. With the present conventions, we have

$$
A=-\left.\frac{\pi^{d / 2} \tilde{L}^{d+1}}{d \Gamma(d / 2)} \mathcal{L}\right|_{A d S} .
$$

While this result was derived for even $d$, one can confirm that same result applies for $a_{d}^{*}$ in odd $d$ with quasi-topological gravity (2.6) or any of the gravitational theories considered in section 3. Hence we will use eq. (5.23) to provide a simple definition for $a_{d}^{*}$ for a general gravitational theory (5.6) in both odd and even dimensions, i.e.,

$$
a_{d}^{*} \equiv-\left.\frac{\pi^{d / 2} \tilde{L}^{d+1}}{d \Gamma(d / 2)} \mathcal{L}\right|_{A d S} .
$$

In this case, the signature of the bulk spacetime does affect the overall sign in this result. Hence in terms of the the Euclidean signature Lagrangian, one finds:

$$
\left.a_{d}^{*} \equiv \frac{\pi^{d / 2} \tilde{L}^{d+1}}{d \Gamma(d / 2)} \mathcal{L}_{\mathrm{E}}\right|_{A d S} .
$$

We have now established our three key results, eqs. (5.14), (5.22) and (5.25). Recall that these all apply for a completely general gravity theory (5.7). Combining these 
equations, we arrive at the following expression for the entanglement entropy

$$
S=\left.\frac{2 \pi}{\pi^{d / 2}} \frac{\Gamma(d / 2)}{\tilde{L}^{d-1}} a_{d}^{*} \int d^{d-1} x \sqrt{h}\right|_{\Sigma_{b}} .
$$

Again we observe that the surface $\Sigma_{b}$ in the Euclidean $\mathrm{AdS}_{d+1}$ geometry coincides with the bifurcation surface of the hyperbolic black hole considered in section 4 upon analytic continuation back to Minkowski signature. Hence our result (5.26) here precisely matches that given in eqs. (4.2) and (4.5). Hence we recover the area law in eq. (4.6) and the universal contribution to the entanglement entropy

$$
S_{\text {univ }}= \begin{cases}(-)^{\frac{d}{2}-1} 4 a_{d}^{*} \log (2 \tilde{L} / \delta) & \text { for even } d, \\ (-)^{\frac{d-1}{2}} 2 \pi a_{d}^{*} & \text { for odd } d .\end{cases}
$$

Here we have established the role of $a_{d}^{*}$ in the holographic entanglement entropy for a completely general bulk theory of gravity. However, in this general context, one cannot expect that $a_{d}^{*}$ always satisfies the holographic c-theorem (2.15). The latter would still require that the gravitational couplings are constrained along the lines discussed in section 3 .

\subsection{Entanglement entropy without holography}

Here we set aside our discussion of holographic entanglement entropy and instead consider a purely field theoretic calculation of entanglement for the configuration considered above. For a conformal field theory in an even number of spacetime dimensions, the universal coefficient in the entanglement entropy can be determined through the trace anomaly. This approach relies on the geometric approach [42] for calculating the entanglement entropy that was discussed at the beginning of this section. The connection to the trace anomaly first appeared in calculations for two-dimensional CFT's [49] - see related ideas in [50] - and later extended to higher dimensions [18, 46]. In the following, our discussion follows closely that presented in [18].

As above, we begin with considering the case of a $d$-dimensional CFT on $R \times S^{d-1}$. Dividing the sphere into two along the equator $\Sigma$, i.e., along a maximal $S^{d-2}$, we wish to determine the universal coefficient in the entanglement entropy between the two halves of the sphere. Recall that with the geometric approach, we must evaluate the partition function on a background geometry with an infinitesimal conical defect. In order to construct a symmetric geometry where introducing such a defect is well-defined, we conformally transform the (Euclidean) background metric (5.3) to a $d$-sphere metric (5.5). Given the rotational symmetry around $\Sigma$ with the latter metric, we construct $\mathcal{M}_{1-\epsilon}$, the ' $n$-fold cover' with $n=1-\epsilon$, by introducing an infinitesimal conical defect 
at $\Sigma$. The expression for the entanglement entropy (5.2) then becomes

$$
S=\lim _{\epsilon \rightarrow 0}\left(\frac{\partial}{\partial \epsilon}+1\right) \log Z_{1-\epsilon}
$$

Now we observe that there is a single scale in the particular geometry for which we are calculating the entanglement entropy, i.e., $\tilde{L}$, the radius of curvature of the original $S^{d-1}$ in (5.3). To connect the calculation to the trace anomaly, we consider shifting this scale with $[18]^{16}$

$$
\begin{aligned}
\tilde{L} \frac{\partial S}{\partial \tilde{L}} & =\lim _{\epsilon \rightarrow 0}\left(\frac{\partial}{\partial \epsilon}+1\right) \tilde{L} \frac{\partial}{\partial \tilde{L}} \log Z_{1-\epsilon} \\
& =\lim _{\epsilon \rightarrow 0}\left(\frac{\partial}{\partial \epsilon}+1\right) \int d^{d} x \sqrt{g}\left\langle T_{a}^{a}\right\rangle \\
& =\lim _{\epsilon \rightarrow 0}\left(\frac{\partial}{\partial \epsilon}+1\right) \int d^{d} x \sqrt{g}\left[\sum B_{i} I_{i}-2(-)^{d / 2} A E_{d}\right] .
\end{aligned}
$$

In the last line, we have used eq. (1.4) to replace the trace of the stress tensor. Now we observe that the resulting expression has essentially the same form as eq. (5.12), in that, we are measuring the linear response to $\epsilon$ of an integral of some scalar constructed with curvatures and covariant derivatives in the background geometry. Hence we can follow the analysis presented in the holographic calculation and we arrive at the following analog of eq. (5.14) for the entanglement entropy

$$
\tilde{L} \frac{\partial S}{\partial \tilde{L}}=2 \pi \int d^{d-2} x \sqrt{h} \tilde{\varepsilon}^{a b} \tilde{\varepsilon}_{c d}\left[\sum B_{i} \frac{\partial I_{i}}{\partial R_{c d}^{a b}}-2(-)^{d / 2} A \frac{\partial E_{d}}{\partial R_{c d}^{a b}}\right]_{\Sigma} .
$$

At this point, we must examine the expressions entering the trace anomaly (1.4) in more detail. Recall that $E_{d}$ is the Euler density in $d$ dimensions and $I_{i}$ are independent Weyl invariants constructed from contractions of $d / 2$ curvatures or $d / 2-k$ curvatures and $2 k$ covariant derivatives. There is some ambiguity in constructing the invariants $I_{i}$, however, a general construction was studied in [51]. In this approach, the natural building blocks of the invariants are the Weyl tensor $W_{a b c d}$, the Cotton tensor $C_{a b c}$ and the Bach tensor $B_{a b}$ (as well as covariant derivatives of these). ${ }^{17}$ Next we observe that

\footnotetext{
${ }^{16}$ For readers following the details here, we emphasize that these calculations are made for a background with Euclidean signature.

${ }^{17}$ As the latter two tensors may be unfamiliar to some readers, we recall that

$$
\begin{aligned}
C_{a b c} & =\nabla_{c} R_{a b}-\nabla_{b} R_{a c}+\frac{1}{2(d-1)}\left(g_{a c} \nabla_{b} R-g_{a b} \nabla_{c} R\right), \\
B_{a b} & =P_{c d} W_{a}^{c}{ }^{d}{ }^{d}+\nabla^{c} \nabla_{a} P_{b c}-\nabla^{2} P_{a b} \quad \text { with } P_{a b}=\frac{1}{d-2}\left(R_{a b}-\frac{R}{2(d-1)} g_{a b}\right) .
\end{aligned}
$$
}


these basis tensors all vanish for the spherical metric (5.5), i.e., $W_{a b c d}=0=C_{a b c}=B_{a b}$ for a round $S^{d}{ }^{18}$ Further with this construction, all of the $I_{i}$ are at least quadratic in these three tensors and as a result, $\partial I_{i} / \partial R^{a b}{ }_{c d}$ is at least linear in one of these tensors. Therefore we can conclude that all of the contributions in eq. (5.30) proportional to the coefficients $B_{i}$ vanish in the present calculation. This leaves us only to consider the contribution from the A-type anomaly.

Our convention for the normalization of the Euler density $E_{d}$ in eq. (1.4) is that on a $d$-dimensional sphere: $\oint_{S^{d}} d^{d} x \sqrt{g} E_{d}=2$. With this normalization, it is straightforward to show that ${ }^{19}$

$$
2 \pi \tilde{\varepsilon}^{a b} \tilde{\varepsilon}_{c d} \frac{\partial E_{d}}{\partial R^{a b}{ }_{c d}}=E_{d-2} .
$$

which we then substitute into eq. (5.30). Recall that the conical singularity lies on a maximal $S^{d-2}$ and hence integrating over this surface, we find the following simple result:

$$
\tilde{L} \frac{\partial S}{\partial \tilde{L}}=(-)^{\frac{d}{2}-1} 4 A .
$$

If we integrate this expression with respect to $\tilde{L}$, we arrive at

$$
S=(-)^{\frac{d}{2}-1} 4 A \log (\tilde{L} / \delta)
$$

where $\delta$ is the short-distance cut-off that we use to regulate the calculations. Hence we see that the coefficient of the universal term in the entanglement entropy is proportional to the central charge $A$. Note that this result (5.34) does not quite match the holographic results for even $d$ presented in eqs. (4.7) and (5.27). However, the difference can be simply regarded as a non-universal constant term.

In general, this approach to calculating the entanglement entropy (with even $d$ ) yields a result depending on all of the central charges appearing in the trace anomaly [46]. Here we have chosen a very specific background geometry and a specific boundary dividing this space and we have found that the universal coefficient in entanglement entropy (5.34) singles out precisely $A$, the central charge of the Euler density in eq.(1.4). This matches our holographic result (5.27) since we have also identified $a_{d}^{*}=A$ for even d. However, eq. (5.34) is a general statement about $S_{\text {univ }}$ on $S^{d-1} \times R$ with any CFT in even $d$.

Let us add a few additional remarks about this calculation: Firstly, the general approach employed above can only be successfully applied when the system for which

\footnotetext{
${ }^{18} \mathrm{As}$ an aside, let us note that simple result which follows from this construction is that the only contribution to the trace anomaly in $d$ dimensions is $A$, i.e., $\oint_{S^{d}}\left\langle T^{a}{ }_{a}\right\rangle=4(-)^{(d-2) / 2} A$.

${ }^{19}$ Here one can use related results for Lovelock gravity in $[36,45]$. Recall, however, that the present calculations are performed for a Euclidean signature.
} 
we are calculating the entanglement entropy contains a single scale. This is certainly

the case for the present case, where the scale $\tilde{L}$ characterizes both the curvature of the background sphere and the size of the equator dividing this $S^{d-1}$ into two halves. Further, the rotational symmetry around the equator of the $d$-sphere ensures that the extrinsic curvature of this surface vanishes. Otherwise we should have expected that additional 'corrections' involving the extrinsic curvature should be added to the result in eq. (5.30) [46]. Also, note that in making use of the trace anomaly in eq. (5.29), the calculation works with the renormalized stress tensor and so we have implicitly discarded divergent terms that might have naturally appeared with inverse powers of $\delta$. Hence the present calculation has eliminated the non-universal terms that might have appeared in the entanglement entropy, e.g., the leading 'area' term. Finally if this calculation was performed in an odd number of spacetime dimensions, the result would vanish because the trace anomaly is zero for odd $d$. However, this is in keeping with the expectation that there is no logarithmic contribution to the entanglement entropy for odd $d$.

\subsection{Counting degrees of freedom}

Ref. [52] considered how the AdS/CFT correspondence realized the 'holographic principle' $[53,54]$. Of course, the latter proposes that in a consistent theory of quantum gravity, the physics within a macroscopic region of space can be described by a dual theory living on the boundary of that region. However, central element of this proposal is that 'the boundary theory should not contain more than one degree of freedom per Planck area.' While the first aspect of the proposal is obviously realized in the AdS/CFT framework, ref. [52] examined the latter for the specific example of the correspondence between $N=4$ super-Yang-Mills (SYM) and Type IIb strings in $\mathrm{AdS}_{5} \times S^{5}$. As the boundary theory is a CFT, it contains an infinite number of degrees of freedom unless one introduces a short distance cut-off, $\delta$. As the SYM theory has a $U\left(N_{c}\right)$ gauge group, then one has roughly $N_{\text {dof }} \simeq N_{c}^{2} V_{3} / \delta^{3}$ in the regulated theory, where $V_{3}$ is the spatial volume. In the $\mathrm{AdS}_{5}$ geometry, the short distance cut-off is associated with a regulator surface at some large radius $R=\tilde{L}^{2} / \delta$. Then using the standard AdS/CFT dictionary, the authors of [52] show

$$
N_{\mathrm{dof}} \simeq N_{c}^{2} \frac{V_{3}}{\delta^{3}} \simeq \frac{\mathcal{A}_{3}(R)}{\ell_{\mathrm{P}}^{3}}
$$

where $\mathcal{A}_{3}(R)$ is the 'area' of (a constant $t$ slice of) the regulator surface. This result then confirms that both of the desired aspects of the holographic principle are realized in this example of the AdS/CFT correspondence. 
Implicitly above, we are considering a regime where the bulk string theory is well approximated by Einstein gravity in $\mathrm{AdS}_{5}$. Then we see in eq. (5.35) that the Bekenstein-Hawking entropy evaluated on a holographic screen at some large $R$ reproduces the counting of the degrees of freedom in the regulated boundary CFT. Let us now apply this reasoning in the more general framework with higher curvature gravity in the bulk that we have been studying. Rather than using the Hawking-Bekenstein entropy here, it is natural to evaluate Wald's entropy formula (4.8) on the holographic screen near the $A d S_{d+1}$ boundary. Then following the reasoning above but in reverse, we expect that this geometric result will count the number of degrees of freedom in the (regulated) boundary theory.

Let us begin with the $\mathrm{AdS}_{d+1}$ metric in global coordinates

$$
d s^{2}=\frac{d r^{2}}{\left(\frac{r^{2}}{\tilde{L}^{2}}+1\right)}-\left(\frac{r^{2}}{\tilde{L}^{2}}+1\right) d t^{2}+r^{2} d \Omega_{d-1}^{2},
$$

where $d \Omega_{d-1}^{2}$ is the metric on a unit $(d-1)$-sphere. Extracting the conformal factor $r^{2} / \tilde{L}^{2}$ from the asymptotic metric along the CFT directions as usual, we arrive at the following metric for the boundary theory:

$$
d s^{2}=-d t^{2}+\tilde{L}^{2} d \Omega_{d-1}^{2}
$$

Next we calculate the Wald entropy on a holographic screen near the boundary. That is, the expression in eq. (4.8) is evaluated on an $S^{d-1}$ at some large radius $R$ (and constant $t$ ). Interpreting the result as the number of degrees of freedom in the boundary theory, we have

$$
N_{\text {dof }}=-\left.2 \pi \frac{\partial \mathcal{L}}{\partial R^{a b}{ }_{c d}} \hat{\varepsilon}^{a b} \hat{\varepsilon}_{c d} \int d^{d-1} x \sqrt{h}\right|_{(r=R, t=\text { constant })},
$$

where the pre-factor above is again constant. Note that, just as in the Wald expression (4.8) for black hole entropy, $\hat{\varepsilon}_{a b}$ is the two-dimensional volume form in the space transverse to the surface on which the entropy is evaluated. Given the metric (5.36), the 'area' integral yields $\int d^{d-1} x \sqrt{h}=R^{d-1} \Omega_{d-1}=(R / \tilde{L})^{d-1} V_{d-1}$ where $V_{d-1}$ is the volume of the corresponding surface in the boundary CFT with eq. (5.37). With the standard general arguments $[52,55]$ applied to our generalized holographic framework, we derive the usual relation between the radius $R$ in $\operatorname{AdS}_{d+1}$ and a short-distance cut-off $\delta$ in the boundary theory, i.e., $R=\tilde{L}^{2} / \delta$. Hence eq. (5.38) can be re-expressed as

$$
\begin{aligned}
N_{\text {dof }} & =-\left.2 \pi\left(\frac{\tilde{L}}{\delta}\right)^{d-1} V_{d-1} \frac{\partial \mathcal{L}}{\partial R^{a b} c d} \hat{\varepsilon}^{a b} \hat{\varepsilon}_{c d}\right|_{(r=R, t=\text { constant })} \\
& =\frac{2 \pi}{\pi^{d / 2}} \Gamma(d / 2) a_{d}^{*} \frac{V_{d-1}}{\delta^{d-1}}
\end{aligned}
$$


Recalling that the background geometry is simply $\mathrm{AdS}_{d+1}$, we have applied eqs. (5.21) and (5.24), as well as $\hat{\varepsilon}^{a b} \hat{\varepsilon}_{a b}=-2$, in producing the final expression.

Hence our expression (5.39) for the number of degrees of freedom is very similar in form to that (5.35) for the $N=4 \mathrm{SYM}$. In particular, the factor of $V_{d-1} / \delta^{d-1}$ counts the number of minimum size cells in regulated boundary theory, as above. Further $a_{d}^{*}$ replaces the factor of $N_{c}^{2}$ in counting the number of degrees of freedom in each such cell. Hence the present calculation suggests that $a_{d}^{*}$ provides a measure of the density of the degrees of freedom in the boundary CFT. Since we are beginning with Wald's formula in eq. (5.38), our result does not technically match the 'one degree of freedom per Planck area' rule but realizes it in spirit. After all, this rule emerged from considerations of the Bekenstein-Hawking entropy of black holes in Einstein gravity and here we are taking the natural generalization to higher curvature theories.

\section{Other central charges}

In sections 4 and 5 , we were able to identify $a_{d}^{*}$ with the universal coefficient in a certain calculation of entanglement entropy. Further, for even $d$, we showed that $a_{d}^{*}$ precisely matches $A$, the central charge multiplying the Euler density in the trace anomaly (1.4). One of the advantages of working with a holographic model where the bulk gravity theory contains higher curvature interactions is that we can distinguish between the different central charges in the dual CFT. Hence we are also able to show that $a_{d}^{*}$ does not agree with various candidate charges that have been considered in examining possible c-theorems in higher dimensions.

One proposal for a c-theorem [24] is to consider the coefficient characterizing the leading singularity in the two-point function of two stress tensors [24, 56]:

$$
\left\langle T_{a b}(x) T_{c d}(0)\right\rangle=\frac{C_{T}}{x^{2 d}} \mathcal{I}_{a b, c d}(x),
$$

where

$$
\mathcal{I}_{a b, c d}(x)=\frac{1}{2}\left(I_{a c}(x) I_{b d}(x)+I_{a d}(x) I_{b c}(x)\right)-\frac{1}{d} \eta_{a b} \eta_{c d}
$$

and

$$
I_{a b}(x)=\eta_{a b}-2 \frac{x_{a} x_{b}}{x^{2}} .
$$

This coefficient $C_{T}$ is a "central charge" common to CFT's in any number of dimensions, $d$. Of course, up to the normalization, $C_{T}$ also corresponds to a standard central charge appearing in the trace anomaly, e.g., $C_{T}=4 c$ in eq. (1.2) for $d=2$ and $C_{T}=\left(40 / \pi^{4}\right) c$ in eq. (1.3) for $d=4$. As such, various counter-examples to this proposed c-theorem exist for four dimensions $[5,6]$ and so it cannot hold in complete generality. In any 
event, it is straightforward to calculate the two-point function (6.1) in the dual gravity theory, e.g., see $[16,26]$, and using the notation of section 3, we find

$$
C_{T}=\frac{d+1}{d-1} \frac{\Gamma[d+1]}{\pi^{d / 2} \Gamma[d / 2]} \frac{\tilde{L}^{d-1}}{\ell_{\mathrm{P}}^{d-1}}\left(1-2 f_{\infty} \hat{\lambda}-3 f_{\infty}^{2} \hat{\mu}\right) .
$$

Here it is clear that $a_{d}^{*}$ and $C_{T}$ are not related (in any simple way) in our holographic models.

In considering extensions of the c-theorem to higher dimensions, another proposal $[57,58]$ was to consider the leading coefficient of the free energy density at finite temperature. Here we define this 'central charge' $C_{S}$ in terms of the thermal entropy density using the fact that $s / T^{d-1}$ provides a dimensionless ratio characterizing a CFT in $d$ dimensions. We adopt the normalization of [59] in defining $C_{S}$ as

$$
C_{S} \equiv \frac{d+1}{d-1}\left(\frac{d}{2 \pi^{3 / 2}}\right)^{d} \frac{\Gamma((d+1) / 2)}{\sqrt{\pi}} \frac{s}{T^{d-1}} .
$$

With this normalization, $C_{S}$ is again related to the usual central charge in two dimensions by a simple numerical factor, i.e., $C_{S}=4 c$. However, there is no simple relation between $C_{S}$ and any other central charges characterizing a strongly coupled CFT in higher dimensions, e.g., see $[59,60]$. We have $C_{S} / C_{T}=1$ for strongly coupled $N=4$ SYM in four dimensions while at weak coupling, the SYM theory yields $C_{S} / C_{T}=4 / 3$ [60] - see also [26] for further discussion. The strong coupling result $C_{S} / C_{T}=1$ applies for any holographic CFT dual to Einstein gravity. Of course, it is derived by studying the thermal behaviour of planar $\mathrm{AdS}_{d+1}$ black holes in the gravity theory. Unfortunately we do not have solutions describing these black holes for the general theories considered in section 3. Hence we focus our attention on quasi-topological gravity studied in section 2 and in this case, the necessary black holes and their thermodynamic properties were studied in [15]. Given the normalization in eq. (6.5), we find

$$
C_{S}=\frac{d+1}{d-1} \frac{\Gamma[d+1]}{\pi^{d / 2} \Gamma[d / 2]} \frac{\tilde{L}^{d-1}}{\ell_{\mathrm{P}}^{d-1}}\left(\frac{f_{\infty}}{\alpha}\right)^{d-1}
$$

where $\alpha$ is the parameter appearing in the cosmological term in the action (2.6). Using eq. (2.9), we can write

$$
\frac{f_{\infty}}{\alpha}=\frac{1}{1-\lambda f_{\infty}-\mu f_{\infty}^{2}} .
$$

Given the expression (6.6) for $C_{S}$, it is again clear that $a_{d}^{*}$ is not related to $C_{S}$ (in any simple way) for our holographic models.

One may further ask whether $a_{d}^{*}$ can be determined by some $n$-point function of the stress tensor, in particular for $d$ odd. Implicitly, such a result already appears 
with $d$ even. There we have shown $a_{d}^{*}=A$ and we know that $A$ can be identified as a particular coefficient in a $(d / 2+1)$-point function [61]. ${ }^{20}$ Hence in $d=2, A$ appears as the coefficient appearing in the two-point function while in $d=4$, it appears in the three-point function. However, for higher (even) dimensions, one must examine higher $n$-point functions to determine $A$. It would be natural/interesting to explore whether a similar story applies for odd dimensions. In particular, it may seem natural to guess that $a_{d}^{*}$ might be identified using the two- and three-point functions for $d=3$. Of course, the analysis above indicates that the coefficient of the two-point function $C_{T}$ and $a_{d}^{*}$ are independent constants. Further our definition (3.27) for $a_{d}^{*}$ in $d=3$ includes a contribution coming from the four-dimensional Euler density in the gravitational action. However, the latter is purely a topological term in this case and so will not effect any equations of motion for gravitational fluctuations. Hence the corresponding coupling constant will not appear in any of the $n$-point functions in the boundary theory suggesting that we cannot reproduce $a_{d}^{*}$ from these correlators. Similar statements apply for our result (3.28) for $a_{d}^{*}$ in $d=5$.

Above, we have shown that in our holographic models, $a_{d}^{*}$ cannot be identified with $C_{T}$ or $C_{S}$. Of course, it could be true that, in our models or in higher dimensional field theories in general, more than one quantity obeys a c-theorem, i.e., an inequality of the form in eq.(2.13). Following the discussion in section 2, one can easily construct flow functions which yield $C_{T}$ or $C_{S}$ in the $\operatorname{AdS}_{d+1}$ vacua. However, examining their behaviour in general RG flow geometries, one cannot readily establish that their radial evolution is monotonic.

However, let us examine the behaviour of $C_{S}$ in a little more detail. ${ }^{21}$ To make progress, let us consider Gauss-Bonnet (GB) gravity, i.e., we set $\mu=0$ in section 2 . In the RG flows, the only quantity that changes is $\alpha$ and so it is more useful to express $C_{S}$ as

$$
C_{S}=\frac{d+1}{d-1} \frac{\Gamma[d+1]}{\pi^{d / 2} \Gamma[d / 2]} \frac{L^{d-1}}{\ell_{\mathrm{P}}^{d-1}} X^{d-1} \quad \text { with } X \equiv \frac{1}{f_{\infty}^{1 / 2}\left(1-\lambda f_{\infty}\right)}
$$

where only the last factor $X^{d-1}$ evolves in the RG flows. Having restricted our attention to GB gravity, eq. (2.9) simplifies to a quadratic equation and we can solve for $f_{\infty}$

$$
f_{\infty}=\frac{1-\sqrt{1-4 \lambda \alpha}}{2 \lambda}
$$

\footnotetext{
${ }^{20}$ The other the central charges $B_{i}$ in the trace anomaly are similarly determined by certain two-, three-, ..., $d / 2$-point functions [61].

${ }^{21}$ We focus on $C_{S}$ since, as commented above, we don't expect $C_{T}$ to obey a c-theorem in general.
} 


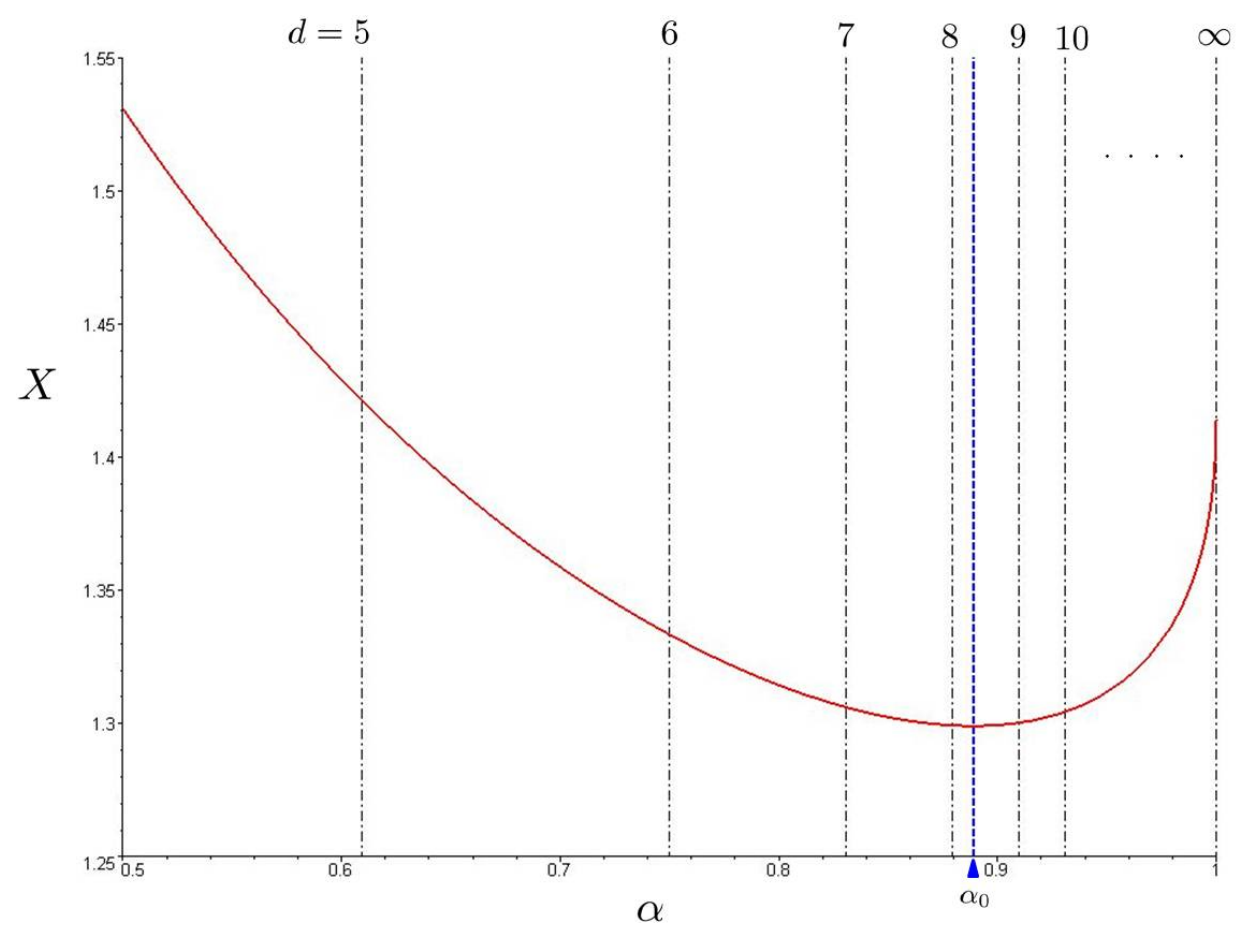

Figure 3: The factor $X$ as a function of $\alpha$ (with $\lambda=1 / 4$ ). The thick blue vertical dashed line indicates $\alpha_{0}$ in eq. (6.10). From left to right, the thin black vertical dashed lines indicate the critical value (6.12) for $d=5,6,7,8,9,10$ and $\infty$. The corresponding line for $d=4$ would appear to beyond the left boundary of the plot, i.e., at $\alpha=0.36$. We see that for $d \geq 9$ there is a regime where flows could yield $\left(C_{S}\right)_{U V}<\left(C_{S}\right)_{I R}$

Using this result (6.9), we plot the result for $X$ in figure 3 , where we see that its slope changes from negative to positive for 'large' values of $\alpha$. In particular, it is a straightforward show that the slope vanishes at

$$
\alpha_{0}=\frac{2}{9 \lambda}
$$

The significance of this observation is that in $\mathrm{RG}$ flows the value of $\alpha$ always increases in going from a UV fixed point to an IR fixed point. Hence any flows in which $\alpha \leq \alpha_{0}$ will always satisfy $\left(C_{S}\right)_{U V}>\left(C_{S}\right)_{I R}$ in this class of models. On the other hand, flows which extend past the critical value $\alpha_{0}$ can violate this inequality.

Now we recall that the analysis of $[25,26]$ shows that in $(d+1)$-dimensional GB theory with fixed $\alpha=1$ there is an upper bound on the gravitational coupling $\lambda$, i.e.,

$$
\lambda \leq \lambda_{c}=\frac{(d-2)(d-3)\left(d^{2}-d+6\right)}{4\left(d^{2}-3 d+6\right)^{2}}
$$


This ensures that the dual field theory was causal and has positive energy fluxes. In the present framework, where $\lambda$ is fixed but $\alpha$ varies, this translates into an upper bound on the latter

$$
\alpha \leq \alpha_{c}=\frac{\lambda_{c}}{\lambda} .
$$

Hence we will not violate the desired c-theorem in this 'physical' regime as long as this critical value is smaller that $\alpha_{0}$. It turns out that this inequality is satisfied for $d \leq 8$ but violated for $d \geq 9$, as illustrated in figure 3 . Hence it seems that we are able to violate the desired c-theorem in this class of holographic models when the spacetime dimension is large.

Note that these violations occur very near the boundary $\alpha=\alpha_{\max }=1 /(4 \lambda)$, beyond which the GB theory has no AdS vacua (or any other maximally symmetric vacuum solutions). That is, the roots of eq. (2.9) with $\mu=0$ are both complex, as can be seen in eq. (6.9). We might note that as $\alpha \rightarrow \alpha_{\max }$, the central charge $C_{T}$ also approaches zero. Further, one finds that the ratio of shear viscosity to entropy density can approach zero at this boundary [27]. These unusual physical properties, as well as the fact that RG flows may yield $\left(C_{S}\right)_{U V}<\left(C_{S}\right)_{I R}$, may indicate that the theory is actually pathological in this regime, although we have no direct evidence of any pathology at this point.

\section{Discussion}

In sections 2 and 3, we have examined RG flows for a broad class of holographic models and found that these theories naturally gave rise to a holographic c-theorem. Further, in sections 4 and 5, we showed that the quantity satisfying the c-theorem, i.e., satisfying eq. (2.15), can be identified with the coefficient of the universal contribution to the entanglement entropy in a particular construction. Our results led us to make the following general conjecture [14]:

- Placing a d-dimensional CFT on $S^{d-1} \times R$ and calculating the entanglement entropy of the ground state between two halves of the sphere, one finds a universal contribution: $S_{\text {univ }} \propto a_{d}^{*}$ (as detailed in eq. (5.27)). Then in $R G$ flows between fixed points, $\left(a_{d}^{*}\right)_{\mathrm{UV}} \geq\left(a_{d}^{*}\right)_{\mathrm{IR}}$.

In particular, our results lend credence to the idea that the universal coefficients appearing in entanglement entropy play the role of central charges in odd-dimensional CFT's. Hence our conjecture above provides a framework to consider the c-theorem for quantum field theories in spacetimes with either an odd or even number of dimensions. 
For the case of even-dimensional boundary theories, we were able to show that the universal coefficient that we identified using the entanglement entropy was precisely the coefficient of the A-type trace anomaly (1.4). In fact, in section 5.4, we were able to show that for any even-dimensional CFT, the universal coefficient appearing the entanglement entropy is precisely the A-type central charge, without any reference to holography. Therefore our conjecture above coincides precisely with Cardy's proposal [7] for any even $d$ and any evidence for Cardy's conjecture also supports the present conjecture. However, the present results also frame Cardy's conjecture in the context of entanglement entropy. This new perspective may provide some useful insights towards constructing a general proof of the c-theorem in higher dimensions. It is quite remarkable, that after more than twenty years, no counter-example is known but a general proof of Cardy's proposal is still lacking.

Of course, entanglement entropy has previously been considered in the context of RG flows and c-theorems. In particular, [62] provided a complementary proof of Zamalodchikov's c-theorem [1] in $d=2$ based on considerations of entanglement entropy, using Lorentz symmetry and the strong subadditivity. One obstacle to generalizing their analysis to higher dimensions was that it was unclear what geometry to specify in calculating the entanglement entropy [62]. In our conjecture, we specify a very particular geometry. This prescription for the geometry was essential to finding $S_{\text {univ }} \propto A$ in even $d$, whereas for a general surface, this coefficient in the entanglement entropy will be given by some linear combination of all of the central charges appearing in the trace anomaly $[18,46]$ - as described in section 5.4. Hence our construction may provide a starting point to extend the results of [62] to higher $d$. It would, of course, be interesting to identify other geometries for which the universal entanglement entropy is proportional to $a_{d}^{*}$. At least for even $d$, one can show that the same universal coefficient appears when the entanglement entropy is calculated across a spherical boundary in flat space [40]. That is, the central charge $A$ controls the entanglement entropy in this geometry as well - a result that was already known for general four-dimensional CFT's [46] and a massless conformally coupled scalar for any even $d[63,64] .^{22}$

Further evidence for Cardy's proposed c-theorem and our conjecture above can be found by examining RG flows induced by double-trace operators [67, 68]. Of course, a greater challenge is to find evidence for the conjectured c-theorem outside of a holographic framework. As explained above, for even $d$, this conjecture coincides with

\footnotetext{
${ }^{22}$ For comparison purposes, the appropriate coefficient for the trace anomaly of a conformally coupled scalar may be found in [65]. We further note that [64] also provides a result for a four-dimensional Maxwell field which disagrees with the general result [46] that $S_{\text {univ }} \propto A$. However, we expect that this can be corrected by accounting for certain subtleties in the vector calculation, as elaborated in $[66]$.
} 
Cardy's proposal [7] and so implicitly any evidence for the latter also supports the present conjecture. Hence it is more interesting to consider the case of odd $d$. We can infer that the entanglement entropy decreases along RG flows for certain known examples in $d=3$ (and 2) [69, 70, 71]. However, we cannot say that these examples provide direct support of our conjecture primarily because the entanglement entropy is not calculated for the geometry specified here.

Here we must acknowledge a technical point with regards to odd $d .{ }^{23}$ Following [18], we have identified the universal term in the entanglement entropy as the constant term appearing in the expansion of eq. (4.5) for large $\rho_{\max }$ or for a small cut-off $\delta=\tilde{L} / \rho_{\max }$. The universality of this constant contribution to the entanglement entropy is wellestablished for a wide variety of $d=3$ conformal quantum critical systems [71], as well as certain three-dimensional (gapped) topological phases [72]. However, in our calculations, it seems that we could replace the cut-off with $\delta^{\prime}=\delta+\delta^{2} / \ell$ where $\ell$ is some macroscopic scale. Such a redefinition of the cut-off would modify the above expansion and in particular change the constant contribution. One objection to such a redefinition of the cut-off should be that at a conformal fixed point, there is no natural macroscopic scale that could play the role of $\ell$. In other words, the only macroscopic length scales would seem to be defined by the geometry of the background or the region in the entanglement entropy calculation, but it seems unnatural that the cut-off would be dependent on these scales. A further observation is that if one could restrict the allowed redefinitions to be of the form $\delta^{\prime}=\delta \times f(\delta / \ell)$ where $f$ is even, then the form of the expansion for the entanglement entropy with odd $d$ would in fact leave the constant contribution invariant. As a final comment, let us add that in field theory, we are used to the idea that the precise value of, say, a gauge coupling constant is scheme dependent. However, once a scheme is fixed, there is no problem in defining the behaviour of the coupling under renormalization group flow. It could be that a similar resolution applies here for our c-theorem for odd dimensions.

Unfortunately we have no conclusive arguments in regard to the above issues at present. Hence we may have to refine further our characterization of $a_{d}^{*}$ in terms of entanglement entropy described in sections 4 and 5. For example, we expect that these issues can be circumvented by considering the mutual information with an appropriate construction [40]. The mutual information is a combination of entanglement entropies given two spatial subsystems: $I(A, B)=S(A)+S(B)-S(A \cup B)$ - for example, see $[73,74]$. In particular, with separate regions, the subtraction ensures that $I(A, B)$ is free of divergences and any ambiguities.

We provided two holographic calculations of the entanglement entropy for the spe-

\footnotetext{
${ }^{23}$ We are grateful to Horacio Casini, Adam Schwimmer, Misha Smolkin and Stefan Theisen for conversations on this point.
} 
cific geometry described above. First in section 4, the entanglement entropy is calculated by relating it to the thermal entropy of the CFT on the hyperbolic plane and at a particular temperature. In section 5, we constructed a holographic calculation based on the replica trick and we reproduced the same results as in the previous section. Both of these calculations are distinct from the standard holographic calculations of entanglement entropy $[17,18]$, which involves finding the area of a minimal surface in the bulk with ends on the appropriate surface in the boundary. In particular, the latter proposal only applies when gravity in the bulk is described by Einstein's theory and so it cannot be used with gravitational theories with higher curvature interactions. Here we emphasize that the analysis in sections 4 and 5 holds for a completely general covariant gravity action and is not restricted to the theories introduced in sections 2 and 3. On the other hand, if we set to zero the coupling constants controlling the higher curvature interactions, the bulk theory reduces to Einstein gravity and our results for the entanglement entropy would match those found using the standard approach. We also note that at present, there is no derivation for this standard proposal for holographic entanglement entropy $[17,18] .{ }^{24}$ Hence our discussion in sections 4 and 5 then provides the first such derivation, albeit for a calculation of entanglement entropy in a particular geometry. In this regard, our results put the standard proposal $[17,18]$ on a firmer footing since we find agreement in the limit of Einstein gravity. Our derivation in section 4 can be further extended to calculations of entanglement entropy across a general spherical boundary [40]. There has also been some other recent progress in understanding holographic entanglement entropy with higher curvature theories in the bulk [77].

An intuitive understanding of the c-theorem leads to an interpretation that the cfunction provides a measure of the number of degrees of freedom of the underlying QFT. Hence the coefficient of the type-A trace anomaly should play this role for CFT's with even $d$ [65]. Our discussion in section 5.5 makes this connection precise for holographic CFT's in eq. (5.39). Of course, this result applies equally well for odd or even $d$ and so $a_{d}^{*}$ still plays exactly the same role in counting the degrees of freedom of the dual CFT in either case. We emphasize that this result applies for any gravitational theory with a covariant action (5.6). It would be interesting to better understand the connection of this role of counting degrees of freedom and the appearance of $a_{d}^{*}$ as a universal coefficient in entanglement entropy. In this regard, it is noteworthy that in the bulk analysis both results rely on Wald's entropy formula (4.8) for black hole horizons. In any event, it is clear that the coefficient $a_{d}^{*}$ will play an important role in general holographic theories.

\footnotetext{
${ }^{24}$ As emphasized in [75], the derivation presented in [76] is flawed and, for example, leads to incorrect results in holographic calculations of Renyi entropies.
} 
At this point, we re-iterate that our results for holographic entanglement entropy in section 5 hold for any bulk theory with a general covariant gravity action. In fact, it is not difficult to extend the approach of section 4 to a completely general gravity theory as well [40]. On the other hand, our results with regards to holographic c-theorems required tuning the couplings in our gravitational action (3.1) in a particular way. Our motivation here was that the resulting holographic model should be physically reasonable, i.e., the dual CFT should be unitary. The reader may wonder why we limited our models not to include only higher curvature interactions beyond the order curvaturecubed? In fact, there is nothing special about this truncation which was made to simply provide a relatively simple framework in which to calculate. It would of course be interesting to confirm that our general approach extends to higher curvature interactions beyond curvature-cubed terms. Some progress in this direction has been made in [79]. Again we wish to emphasize that our gravity theories should be regarded as toy models which allow us to explore of the role of higher curvature terms in holography. These gravity actions do not obviously emerge from any string theory calculations. One could consider a 'perturbative' limit of our analysis. That is, our results would still carry over in a limit where $\lambda_{i}=O\left(\ell_{\mathrm{P}}^{2} / L^{2}\right)$ and $\mu_{i}=O\left(\ell_{\mathrm{P}}^{4} / L^{4}\right)$, as is done in most string theory calculations. The only caveat then would be that one expects that string theory to also generate higher derivative interactions in the matter sector. Although it may be possible to eliminate all or some of these with field redefinitions [80], in general it is not clear what would replace the null energy condition in this context - we return to this discussion below. A long-term goal remains to better develop our ability to calculate within a string theory framework in order study interesting holographic backgrounds with large curvatures and the corresponding RG flows.

Our construction of physically reasonable holographic models in section 3 focussed on the idea that the boundary theory should be unitary. That is, we tuned the coupling constants for the higher curvature interactions to ensure the the linearized graviton equations are only second order in derivatives. This tuning eliminates the appearance of ghost modes in the bulk theory and of non-unitary operators in the boundary QFT. That such tuning is possible for AdS backgrounds was originally observed for quasitopological gravity in [15] and later in lower dimensional theories as well [32, 33, 30] - see also the discussion in [78]. Again, this approach can also be extended to higher dimensions with higher curvature interactions beyond curvature-cubed terms [79]. For the present analysis, we demanded that this property apply for general RG flows, i.e., for fluctuations around the general metric (2.2), and restricted our general theory (3.1) with eleven coupling constants down to a model with only four independent couplings. Having imposed the unitarity constraints (3.11-3.16), the resulting model automatically satisfied a holographic c-theorem. It would be interesting to see if this result extends 
to more general theories, e.g., with higher curvature terms beyond $R^{3}{ }^{25}$

In this analysis, a key property of the RG flow geometries (2.2) seems to be that they are conformally flat. Of course, there are many physically interesting geometries (e.g., general AdS black hole solutions) which will not share this properties and in these backgrounds, higher derivative terms will reappear in the linearized graviton equations. For example, in quasi-topological gravity, the four-derivative contributions can be expressed as couplings with the background Weyl curvature [15]

$$
W^{c d e f} h_{d e ; c f(a b)}+2\left(\square h_{(a)}^{c}\right) ; d e W_{|c d e| b)}+2 \square^{2} h^{c d} W_{c a d b}+g_{a b}\left(\square h_{c d}\right)_{; e f} W^{c e d f} .
$$

For simplicity, we are only considering transverse traceless modes here (i.e., $\nabla^{a} h_{a b}=$ $\left.0=h^{a}{ }_{a}\right){ }^{26}$ Hence in a background where $W_{a b c d} \neq 0$, the analysis of the linearized fluctuations becomes far more complicated, e.g., see [16].

Let us make two observations at this point: First of all, any asymptotically AdS background describing a scenario where the boundary theory is Poincaré invariant will have a form which matches the metric ansatz (2.2), which we used to consider general RG flows. Hence our analysis in section 3 ensures that no higher derivatives arise for such a Poincaré invariant background. The implication is then that higher derivative terms can only arise when the boundary theory or boundary state is not Lorentz invariant. Therefore the appearance of such terms need not immediately imply the existence of ghost modes. Of course, theories with a Lifshitz symmetry provide simple examples where higher derivative equations of motion do not imply the appearance of ghosts $\left(e . g .,\left(\partial_{t}^{2}+\kappa \nabla^{4}\right) \phi=0\right)$.

Another interesting aspect of the higher derivative terms, such as those illustrated in eq. (7.1), is that they will vanish in the asymptotic region with AdS boundary conditions, since the Weyl curvature will vanish there. Hence any new modes associated with these terms (which are potentially unstable or ghost-like) will be 'confined' to the interior of the geometry. Hence it seems that such modes will not be associated with the appearance of new operators in the boundary theory. Rather such modes would reflect some complicated new infrared dynamics which would be insensitive to the ultraviolet details of the theory. We recall that despite the fact that the full equations of motion of quasi-topological gravity are fourth order in derivatives, the most general static black hole solutions are characterized by a single integration constant [15] - just as in Einstein (or Lovelock) gravity. This result is likely related to this idea that the fourderivative contributions in the linearized equations have no effect near the asymptotic

\footnotetext{
${ }^{25}$ Recall that no three-dimensional gravity theory can constructed with only curvature-squared and curvature-cubed interactions which simultaneously satisfies a c-theorem and is 'unitary' [32].

${ }^{26}$ In eq. (7.1), we have also adopted the standard notation: $T_{(a b)}=\frac{1}{2}\left(T_{a b}+T_{b a}\right)$.
} 
boundary. To properly understand these issues, it seems that one should analyse the asymptotic geometry of a general solution of our 'unitary' higher curvature theories using a Fefferman-Graham-like expansion [51], e.g., following [20].

Our final comment in this regard is that our restriction to second-order linearized equations is sufficient but not necessary to ensure unitarity of the boundary theory. In particular, consider restricting the action (3.1) to contain only the terms containing the Ricci scalar. The resulting equations of motion are indeed fourth order in derivatives but it is well know that such an $f(R)$ theory is equivalent to a theory of Einstein gravity coupled to a 'ordinary' scalar field, e.g., see [81]. If one considers the fourth order linearized equations, one would find that the only extra modes correspond to a massive scalar whose propagator comes with a positive sign, i.e., these modes are not ghost-like - for example, see $[82,83]$. Hence in the AdS/CFT framework, the metric fluctuations couple to a new unitary scalar operator, as well as the stress tensor, in the boundary theory. It would be interesting to do a more general analysis of the gravity equations of motion for the action (3.1) to understand precisely which of the constraints in section 3 can be relaxed while still preserving unitarity in boundary theory.

In constructing our holographic models in sections 2 and 3, we have introduced an unconventional gravity theory, i.e., a higher curvature theory, and a conventional matter theory. Both sectors were constrained in different ways. That is, as we have just discussed, the gravitation couplings were tuned to ensure that no non-unitary operators appear in the dual QFT, while the matter sector was required to satisfy the null energy condition. One interesting question is to better understand the holographic interpretation of the null energy condition in terms of the boundary theory. However, it would also be more interesting to consider more general couplings between the gravitational and matter sectors. For example, if the matter sector was simply a scalar with an interesting potential, one can imagine that rather than having constant gravitational couplings that these would be replaced by functions of the scalar field. That is, we would introduce interactions of the form $\mathcal{W}_{1}(\phi) R^{2}$. Enforcing 'unitarity' constraints extends to the generalized theory in an obvious way, but will now involve linearized equations of motion for both the metric and scalar fields. However, it is not clear what constraints should replace the null energy condition to ensure a holographic c-theorem. Some preliminary analysis of RG flows in the presence of such generalized couplings are presented in appendix B. A full understanding of these issues seems to be a challenging problem which we leave to future work.

Another interesting direction for the future would be to investigate holographic c-theorems for models with non-relativistic symmetries. Models with Schrödinger [84] and Lifshitz [85] symmetries have been studied and further the contribution of higher curvature terms has also been considered in this context [86]. Here we propose to 
consider RG flows between different vacua of such a model. In particular, it would be interesting to consider flows where the symmetry group changes between the UV and IR fixed points. Again an initial investigation of some of these questions in given in appendix $\mathrm{C}$ but a full examination is left to the future.

Acknowledgments: We thank John Cardy, Horacio Casini, Sumit Das, Stuart Dowker, Ben Freivogel, Rajesh Gopakumar, Matt Headrick, Janet Hung, Dileep Jatkar, André LeClair, Shiraz Minwalla, Miguel Paulos, Adam Schwimmer, Al Shapere, Misha Smolkin, Brian Swingle, Lenny Susskind, Yuji Tachikawa, Stefan Theisen and Toby Wiseman for useful discussions. AS thanks the Physics Departments of the University of Kentucky and Princeton University where part of this work was presented. Research at Perimeter Institute is supported by the Government of Canada through Industry Canada and by the Province of Ontario through the Ministry of Research \& Innovation. RCM also acknowledges support from an NSERC Discovery grant and funding from the Canadian Institute for Advanced Research. RCM thanks the Galileo Galilei Institute for Theoretical Physics for hospitality and the INFN for partial support during the completion of this work.

\section{A. RG flows}

In this appendix, we illustrate the holographic RG flows in more detail. For concreteness, we consider the quasi-topological theory with $d=4$ as discussed in section 2 . Recall that the curvature in the $\mathrm{AdS}_{5}$ vacua is set by

$$
\frac{1}{\tilde{L}^{2}}=\frac{f_{\infty}}{L^{2}}, \quad \alpha=f_{\infty}-\lambda f_{\infty}^{2}-\mu f_{\infty}^{3} .
$$

As explained in section 2, we can imagine that the gravity theory is coupled to, e.g., a scalar field with an interesting potential which yields various stationary points. These different stationary points will be distinguished by different values of the (negative) cosmological constant, i.e., they yield different values for the parameter $\alpha$ above. As discussed, we consider the root of eq. (A.1) that is smoothly connected to $f_{\infty}=\alpha$ in the limit $\lambda, \mu \rightarrow 0$.

At each of the stationary points, there are three dimensionless parameters which characterize the gravitational theory, the couplings $\lambda$ and $\mu$ as well as the ratio $\tilde{L} / \ell_{\mathrm{P}}$. Similarly, the dual CFT at the corresponding fixed points can be characterized in 
terms of three parameters defining the three-point function of the stress tensor [24]. Alternatively, these parameters define various other constants which define the dual CFT, e.g., the central charge $a$. Two other convenient constants are $t_{2}$ and $t_{4}$, which arise in certain gedanken experiments described in [87]. In our holographic framework, these two parameters are given by [16]: ${ }^{27}$

$$
t_{2}=\frac{24 f_{\infty}\left(\lambda-87 f_{\infty} \mu\right)}{1-2 \lambda f_{\infty}-3 \mu f_{\infty}^{2}}, \quad t_{4}=\frac{3780 \mu f_{\infty}^{2}}{1-2 \lambda f_{\infty}-3 \mu f_{\infty}^{2}}
$$

In figure 4 , we plot $t_{4}$ against $t_{2}$ for various models. The contours are generated by varying $\alpha$ while keeping the couplings $\lambda$ and $\mu$ fixed. These lines should not be thought of as literal RG flows in parameter space, i.e., $t_{2}$ and $t_{4}$ are only defined at the fixed points. Rather any RG flow in this model will connect two fixed points on the same contour. Note that the origin, i.e., $t_{2}=0=t_{4}$, corresponds to Einstein gravity and the $\mathrm{RG}$ flows generally move out into the plane away from this point. Note however that the RG flows never cross the lines $\mu=0$ or $\lambda=0$, which correspond to $t_{4}=0$ and $t_{2}+\frac{58}{105} t_{4}=0$, respectively. The gedanken experiments defining $t_{2}$ and $t_{4}$ also establish that the corresponding CFT will only be consistent within a (small) region around the origin [87]. The boundaries of this physical region are indicated as the 'flux constraints' in red in figure 4. It would be straightforward to construct the holographic RG flows which pass out through these boundaries. Hence it appears the RG flows are not a refined enough probe of the holographic model to detect the inconsistencies arising in the unphysical region. However, we note that there can be no such flows which enter into the physical region from outside.

To conclude this discussion, however, we must say that we cannot be certain at this point which, if any, of the detailed aspects of the RG flows noted above can be regarded as universal properties. That is, which properties apply outside of the particular class of holographic models based on quasi-topological gravity (2.6).

\section{B. More general couplings}

In this section, we initiate the study of c-theorems with more general couplings between the gravitational sector and the matter fields in the bulk. To be concrete, let us consider the following action

$$
I=\frac{1}{2 \ell_{\mathrm{P}}^{3}} \int d^{5} x \sqrt{-g}\left[\frac{12}{L^{2}} \mathcal{V}(\phi)+R-\frac{1}{2} \partial_{\mu} \phi \partial^{\mu} \phi+\frac{L^{2}}{2} \mathcal{W}(\phi) \chi_{4}\right]
$$

\footnotetext{
${ }^{27}$ These formulae were originally derived in [16] with $\alpha=1$ but the results remain unchanged when one allows $\alpha$ to take on different values in eq. (A.1).
} 


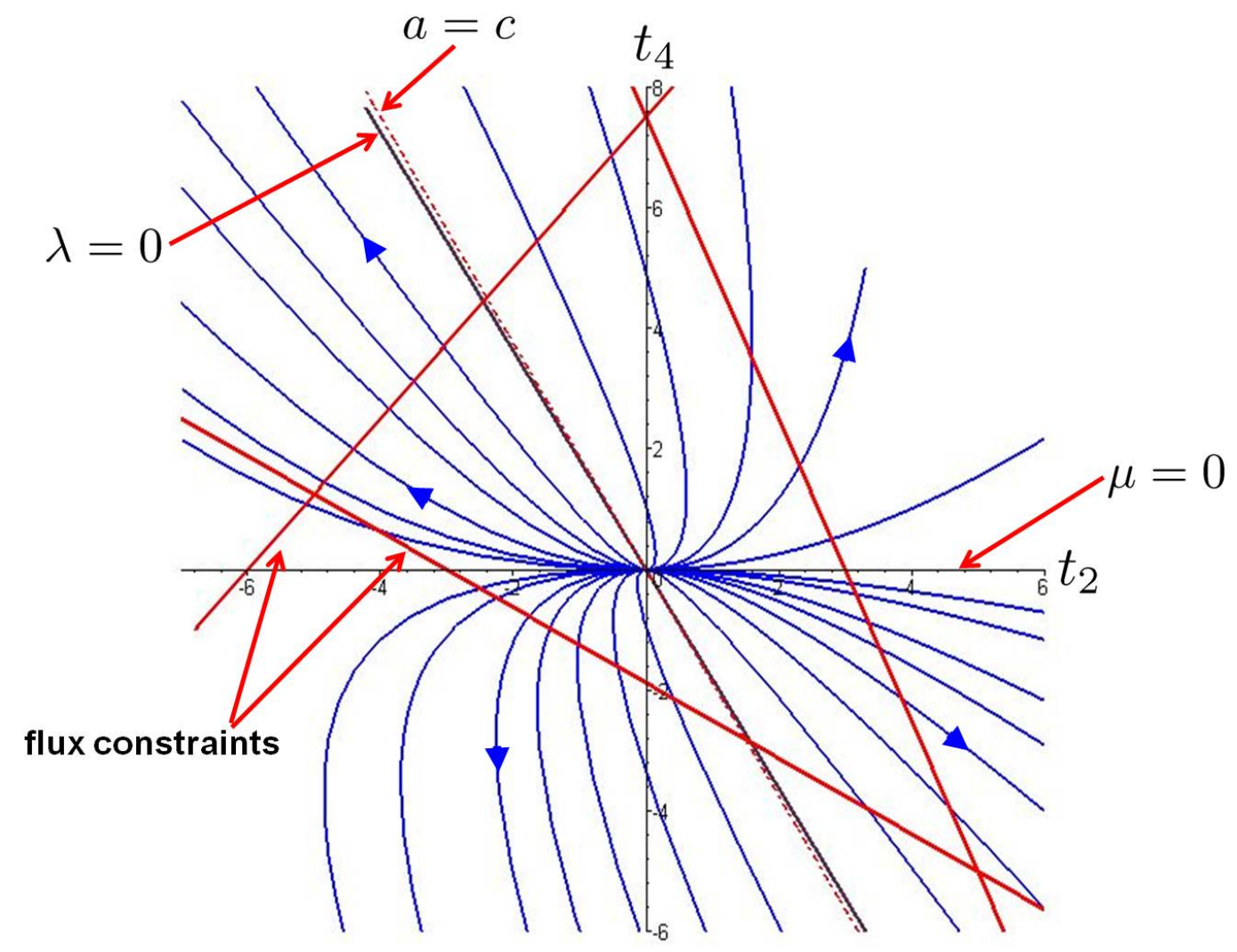

Figure 4: RG flows in the $\left(t_{2}, t_{4}\right)$ plane. Holographic RG flows (in the model described in the main text) connect fixed points on the same contour. The arrows indicate the direction of the flows towards the infrared. The black line indicates $\lambda=0$. No trajectories cross this line or the line $\mu=0$. The dashed red line indicates $a=c\left(\right.$ or $\left.t_{2}+\frac{8}{15} t_{4}=0\right)$ and in principle, it is possible to construct holographic RG flows which cross this line.

where $\chi_{4}$ is the combination of curvature-squared terms corresponding to the fourdimensional Euler density given in eq. (2.7). Above, we have explicitly coupled gravity to a scalar field with a standard kinetic term and a potential $V(\phi)=-12 \mathcal{V}(\phi) / L^{2}$. The new feature of this theory is that we have also introduced a scalar coupling $\mathcal{W}(\phi)$ to the curvature-squared interaction. We might note that despite this new coupling, the equations of motion derived from the above action (B.1) are purely second order in derivatives for any arbitrary background [88] and hence the dual theory is 'unitary', according to the discussion given in section 3 .

As in the main text, we assume that this action yields several stationary points where the scalar is constant, i.e., $\phi=\phi_{i}$, and the bulk geometry is simply $\mathrm{AdS}_{5}$. Recall that the latter corresponds to $A(r)=r / \tilde{L}$ in flow metric (2.2). If we again adopt the notation that the AdS curvature scale is written as $\tilde{L}^{2}=L^{2} / f_{\infty}$, then eq. (2.9) is 
replaced by

$$
\mathcal{V}\left(\phi_{i}\right)=f_{\infty}-\mathcal{W}\left(\phi_{i}\right) f_{\infty}^{2}
$$

Note that since $\chi_{4}$ is nonvanishing in the $\mathrm{AdS}_{5}$ background, the stationary points for the scalar field depends on both $\mathcal{V}$ and $\mathcal{W}$, as well as $f_{\infty}$ (i.e., the curvature). That is, the critical values $\phi_{i}$ must satisfy

$$
\left[\frac{\delta \mathcal{V}}{\delta \phi}+10 f_{\infty}^{2} \frac{\delta \mathcal{W}}{\delta \phi}\right]_{\phi=\phi_{i}}=0
$$

These equations determining the vacuum solutions already illustrate that there is no clean separation between the matter and the gravity sectors with this new action (B.1).

Further, given the lack of such a separation, there is no clear role of the standard null energy condition nor is it clear what rule might replace this condition. Hence we must look elsewhere to construct a flow function for the new theory. Motivated by a possible connection between the flow function and Wald's formula (4.8), we consider

$$
a(r)=\frac{\pi^{2}}{\ell_{\mathrm{P}}^{3} A^{\prime}(r)^{3}}\left[1-6 L^{2} A^{\prime}(r)^{2} \mathcal{W}(\phi)\right] .
$$

That is, we have constructed this function to satisfy eq. (3.26). Now, using the equations of motion, we find

$$
a^{\prime}(r)=\frac{\pi^{2}}{\ell_{\mathrm{P}}^{3} A^{\prime}(r)^{4}}\left[\phi^{\prime}(r)^{2}-6 L^{2} \partial_{r}\left(A^{\prime}(r)^{2} \partial_{r} \mathcal{W}\right)\right] .
$$

In main text, we would have had $\mathcal{W}$ being simply a constant coupling in which case the second term vanishes making the right-hand side clearly positive and hence we would recover the monotonic flow discussed there. However, in eq. (B.5), the right-hand side does not have a definite sign. A necessary and sufficient condition for $a(r)$ to grow monotonically is

$$
6 L^{2} \partial_{r}\left(A^{\prime}(r)^{2} \partial_{r} \mathcal{W}\right) \leq \phi^{\prime}(r)^{2}
$$

One might also consider a simpler sufficient (but not necessary) condition

$$
\partial_{r}\left(A^{\prime}(r)^{2} \partial_{r} \mathcal{W}\right) \leq 0
$$

However, if we demand that the end-points of the RG flow are CFTs, i.e., the bulk geometry is $\mathrm{AdS}_{5}$, then $\phi^{\prime}$ vanishes there and so we also have $\partial_{r} \mathcal{W}=\delta \mathcal{W} / \delta \phi \phi^{\prime}=0$ at both end points. Of course, this means that if derivative in eq. (B.7) is anywhere negative, this must be compensated by it being positive elsewhere along the flow. Therefore the only circumstance to in which eq. (B.7) is satisfied is if $\mathcal{W}$ is constant. 
It may be that a more detailed investigation of RG flows for this theory would reveal that in fact, the condition (B.6) is always satisfied, at least if certain conditions are imposed on $\mathcal{W}$ and $\mathcal{V}$. One could start such an investigation by constructing explicit flow solutions with concrete choices of $\mathcal{V}$ and $\mathcal{W}$.

If eq. (B.6) is satisfied for a broad class of models, we expect that there should a better choice of the flow function for which the radial derivative becomes manifestly positive. We did try to modify our expression (B.4) above in various ways but we were unable to find a new flow function which clearly grows monotonically in the radial direction. One such attempt was to consider the following function

$$
\tilde{a}(r)=\frac{\pi^{2}}{\ell_{\mathrm{P}}^{3} A^{\prime 3}}\left[1-6 L^{2} A^{\prime 2} \mathcal{W}(\phi)-6 L^{2} A^{\prime} \frac{\delta \mathcal{W}}{\delta \phi} \phi^{\prime}\right] .
$$

Note that the new term added above will vanish at the $\mathrm{AdS}_{5}$ fixed points and so the new flow function and that in eq. (B.4) will yield identical results when evaluated at the fixed points. In this case, using the equations of motion, the radial derivative becomes

$$
\tilde{a}^{\prime}(r)=\frac{\pi^{2}}{\ell_{\mathrm{P}}^{3} A^{\prime 4}}\left[\phi^{\prime 2}+12 L^{2} \frac{\delta \mathcal{W}}{\delta \phi} \phi^{\prime}\left(A^{\prime 2}\right)^{\prime}\right] .
$$

Unfortunately the result on the right-hand side is again not obviously positive.

It would also be interesting to examine the RG flows of these generalized holographic models along the lines discussed in the previous appendix.

\section{Non-relativistic $c$-theorems}

There has been some interest in holographic models for non-relativistic CFT's, in particular, with Schrödinger symmetry [84]. It is relatively easy to extend our construction of a holographic $c$-theorem to such a framework but the following should only be regarded as an initial step in this direction. Let us consider Gauss-Bonnet gravity in five bulk dimensions, i.e., eq. (2.6) with $d=4$ and $\mu=0$. With the non-relativistic Schrödinger symmetry in mind, we consider the following metric ansatz

$$
d s^{2}=d r^{2}-e^{2 A(r)} d t^{2}+2 e^{2 B(r)} d t d x+e^{2 B(r)} d y^{2}+e^{2 B(r)} d z^{2} .
$$

The usual holographic Schrödinger geometry [84] is recovered with $A(r)=z r / L$ and $B(r) \sim r / L$, where $z$ is the dynamical exponent.

Now let us consider holographic RG flows in this context. If we assume that $T_{t}^{t}-T_{r}^{r} \leq 0$ or equivalently $T_{t x} e^{-2 B}-T_{r r} \leq 0$ then a simple c-theorem can be easily 
proved. Note that this condition does not arise from the null energy condition unless $T_{t t}=0$. In this case, the equations of motion yield

$$
3 B^{\prime \prime}(r)\left(1-2 \lambda L^{2} B^{\prime}(r)^{2}\right)=T_{t}^{t}-T_{r}^{r}
$$

and so that the flow function

$$
a(r)=\frac{1}{B^{\prime}(r)^{3}}\left(1-6 \lambda L^{2} B^{\prime}(r)^{2}\right),
$$

grows monotonically with radius. Alternatively, of course, we have that $a(r)$ is monotonically decreasing along RG flows. Using the null energy condition, we can make one further useful statement. ${ }^{28}$ Consider the following null vector in the $(t, x)$-plane: $v^{a}=\left(0, e^{-A}, 1 / 2 e^{-2 B+A}, 0,0\right)$. The null energy condition then requires

$$
T_{t t} e^{-2 A}+T_{t x} e^{-2 B}+\frac{1}{4} T_{x x} e^{-4 B+2 A} \geq 0 .
$$

Now at the end points of the RG flow, we expect $A(r)$ and $B(r)$ take the form noted above for a Schrödinger geometry. Using this, we derived the following inequality for the dynamical exponents at the UV and IR fixed points:

$$
\left(z_{\mathrm{UV}}^{2}-1\right)\left(1-2 \lambda \frac{L^{2}}{\tilde{L}_{\mathrm{UV}}^{2}}\right) \geq\left(z_{\mathrm{IR}}^{2}-1\right)\left(1-2 \lambda \frac{L^{2}}{\tilde{L}_{\mathrm{IR}}^{2}}\right) \frac{\tilde{L}_{\mathrm{UV}}^{4}}{\tilde{L}_{\mathrm{IR}}^{4}} .
$$

For $\lambda=0$, i.e., Einstein gravity in the bulk, if we assume that $\tilde{L}_{\mathrm{UV}}>\tilde{L}_{\mathrm{IR}}$, then this inequality demands that $z_{\mathrm{UV}} \geq z_{\mathrm{IR}}$. That is, the dynamical exponent is always smaller at the infrared fixed point. It would be interesting to have a better insight into this inequality (C.5) when $\lambda \neq 0$ and also to investigate these RG flows in more detail.

\section{References}

[1] A. B. Zamolodchikov, "Irreversibility of the Flux of the Renormalization Group in a 2D Field Theory," JETP Lett. 43, 730 (1986) [Pisma Zh. Eksp. Teor. Fiz. 43, 565 (1986)].

[2] See, for example:

M. J. Duff, "Observations On Conformal Anomalies," Nucl. Phys. B 125, 334 (1977);

M. J. Duff, "Twenty years of the Weyl anomaly," Class. Quant. Grav. 11, 1387 (1994) [arXiv:hep-th/9308075].

${ }^{28}$ The null-energy condition has been considered before in [89] in the context of non-relativistic symmetries. There it was shown that with a Lifshitz symmetry, $z \leq 1$ is necessary for this condition to hold. 
[3] L. Bonora, P. Pasti and M. Bregola, "Weyl Cocycles," Class. Quant. Grav. 3, 635 (1986).

[4] A. Cappelli, J. I. Latorre and X. Vilasis-Cardona, "Renormalization Group Patterns and C-Theorem in More Than Two Dimensions," Nucl. Phys. B 376, 510 (1992) [arXiv:hep-th/9109041].

[5] A. Cappelli, D. Friedan and J. I. Latorre, "C Theorem And Spectral Representation," Nucl. Phys. B 352, 616 (1991).

[6] D. Anselmi, D.Z. Freedman, M.T. Grisaru and A.A. Johansen, "Nonperturbative formulas for central functions of supersymmetric gauge theories," Nucl. Phys. B 526, 543 (1998) [arXiv:hep-th/9708042];

D. Anselmi, J. Erlich, D.Z. Freedman and A.A. Johansen, "Positivity constraints on anomalies in supersymmetric gauge theories," Phys. Rev. D 57, 7570 (1998) [arXiv:hep-th/9711035].

[7] J. L. Cardy, "Is There a c Theorem in Four-Dimensions?," Phys. Lett. B 215, 749 (1988).

[8] H. Osborn, "Derivation of a Four-Dimensional c-theorem," Phys. Lett. B 222, 97 (1989).

[9] K. A. Intriligator and B. Wecht, "The exact superconformal R-symmetry maximizes a," Nucl. Phys. B 667, 183 (2003) [arXiv:hep-th/0304128];

E. Barnes, K. A. Intriligator, B. Wecht and J. Wright, "Evidence for the strongest version of the $4 \mathrm{~d}$ a-theorem, via a-maximization along RG flows," Nucl. Phys. B 702, 131 (2004) [arXiv:hep-th/0408156].

[10] A.D. Shapere and Y. Tachikawa, "A counterexample to the 'a-theorem'," JHEP 0812, 020 (2008) [arXiv:0809.3238 [hep-th]].

[11] D. Gaiotto, N. Seiberg and Y. Tachikawa, "Comments on scaling limits of 4d N=2 theories," arXiv:1011.4568 [hep-th].

[12] D.Z. Freedman, S.S. Gubser, K. Pilch and N.P. Warner, "Renormalization group flows from holography supersymmetry and a c-theorem," Adv. Theor. Math. Phys. 3, 363 (1999) [arXiv:hep-th/9904017].

[13] L. Girardello, M. Petrini, M. Porrati and A. Zaffaroni, "Novel local CFT and exact results on perturbations of $\mathrm{N}=4$ super Yang-Mills from AdS dynamics," JHEP 9812, 022 (1998) [arXiv:hep-th/9810126];

L. Girardello, M. Petrini, M. Porrati and A. Zaffaroni, Nucl. Phys. B 569, 451 (2000) [arXiv:hep-th/9909047]. 
[14] R. C. Myers and A. Sinha, "Seeing a c-theorem with holography," Phys. Rev. D 82, 046006 (2010) [arXiv:1006.1263 [hep-th]].

[15] R. C. Myers and B. Robinson, "Black Holes in Quasi-topological Gravity," JHEP 1008, 067 (2010) [arXiv:1003.5357 [gr-qc]].

[16] R. C. Myers, M. F. Paulos and A. Sinha, "Holographic studies of quasi-topological gravity," JHEP 1008, 035 (2010) [arXiv:1004.2055 [hep-th]].

[17] S. Ryu and T. Takayanagi, 'Holographic derivation of entanglement entropy from AdS/CFT," Phys. Rev. Lett. 96, 181602 (2006) [arXiv:hep-th/0603001].

[18] S. Ryu and T. Takayanagi, "Aspects of holographic entanglement entropy," JHEP 0608, 045 (2006) [arXiv:hep-th/0605073].

[19] See, for example: S.W. Hawking and G.F.R. Ellis, The Large scale structure of space-time, (Cambridge University Press, Cambridge, 1973).

[20] M. Henningson and K. Skenderis, "The holographic Weyl anomaly," JHEP 9807, 023 (1998) [arXiv:hep-th/9806087];

M. Henningson and K. Skenderis, "Holography and the Weyl anomaly," Fortsch. Phys. 48, 125 (2000) [arXiv:hep-th/9812032].

[21] S. Nojiri and S.D. Odintsov, "On the conformal anomaly from higher derivative gravity in AdS/CFT correspondence," Int. J. Mod. Phys. A 15, 413 (2000) [arXiv:hep-th/9903033];

M. Blau, K.S. Narain and E. Gava, "On subleading contributions to the AdS/CFT trace anomaly," JHEP 9909, 018 (1999) [arXiv:hep-th/9904179].

[22] D. Lovelock, "The Einstein tensor and its generalizations," J. Math. Phys. 12, 498 (1971);

D. Lovelock, "Divergence-free tensorial concomitants," Aequationes Math. 4, 127 (1970).

[23] J. Oliva and S. Ray, "A new cubic theory of gravity in five dimensions: Black hole, Birkhoff's theorem and C-function," Class. Quant. Grav. 27, 225002 (2010) [arXiv:1003.4773 [gr-qc]]; J. Oliva and S. Ray, "A Classification of Six Derivative Lagrangians of Gravity and Static Spherically Symmetric Solutions," arXiv:1004.0737 [gr-qc].

[24] See, for example: H. Osborn and A.C. Petkou, "Implications of Conformal Invariance in Field Theories for General Dimensions," Annals Phys. 231, 311 (1994) [arXiv:hep-th/9307010]; 
J. Erdmenger and H. Osborn, "Conserved currents and the energy-momentum tensor in conformally invariant theories for general dimensions," Nucl. Phys. B 483, 431 (1997) [arXiv:hep-th/9605009].

[25] M. Brigante, H. Liu, R. C. Myers, S. Shenker and S. Yaida, "Viscosity Bound Violation in Higher Derivative Gravity," Phys. Rev. D 77, 126006 (2008) [arXiv:0712.0805 [hep-th]];

A. Buchel and R. C. Myers, "Causality of Holographic Hydrodynamics," JHEP 0908, 016 (2009) [arXiv:0906.2922 [hep-th]];

J. de Boer, M. Kulaxizi and A. Parnachev, "AdS $7 / \mathrm{CFT}_{6}$, Gauss-Bonnet Gravity, and Viscosity Bound," JHEP 1003, 087 (2010) [arXiv:0910.5347 [hep-th]];

X. O. Camanho and J. D. Edelstein, "Causality constraints in AdS/CFT from conformal collider physics and Gauss-Bonnet gravity," JHEP 1004, 007 (2010) [arXiv:0911.3160 [hep-th]];

J. de Boer, M. Kulaxizi and A. Parnachev, "Holographic Lovelock Gravities and Black Holes," JHEP 1006, 008 (2010) [arXiv:0912.1877 [hep-th]];

D. M. Hofman, "Higher Derivative Gravity, Causality and Positivity of Energy in a UV complete QFT," Nucl. Phys. B 823, 174 (2009) [arXiv:0907.1625 [hep-th]];

X. O. Camanho and J. D. Edelstein, "Causality in AdS/CFT and Lovelock theory," JHEP 1006, 099 (2010) [arXiv:0912.1944 [hep-th]].

[26] A. Buchel, J. Escobedo, R. C. Myers, M. F. Paulos, A. Sinha and M. Smolkin, "Holographic GB gravity in arbitrary dimensions," JHEP 1003, 111 (2010) [arXiv:0911.4257 [hep-th]].

[27] X. O. Camanho, J. D. Edelstein and M. F. Paulos, "Lovelock theories, holography and the fate of the viscosity bound," arXiv:1010.1682 [hep-th].

[28] R. V. Buniy, S. D. H. Hsu and B. M. Murray, "The null energy condition and instability," Phys. Rev. D 74, 063518 (2006) [arXiv:hep-th/0606091];

S. Dubovsky, T. Gregoire, A. Nicolis and R. Rattazzi, "Null energy condition and superluminal propagation," JHEP 0603, 025 (2006) [arXiv:hep-th/0512260].

[29] C. Imbimbo, A. Schwimmer, S. Theisen and S. Yankielowicz, "Diffeomorphisms and holographic anomalies," Class. Quant. Grav. 17, 1129 (2000) [arXiv:hep-th/9910267];

A. Schwimmer and S. Theisen, "Entanglement Entropy, Trace Anomalies and Holography," Nucl. Phys. B 801, 1 (2008) [arXiv:0802.1017 [hep-th]].

[30] A. Sinha, "On higher derivative gravity, c-theorems and cosmology," arXiv:1008.4315 [hep-th].

[31] E. Witten, "Anti-de Sitter space and holography," Adv. Theor. Math. Phys. 2, 253 (1998) [arXiv:hep-th/9802150]. 
[32] A. Sinha, "On the new massive gravity and AdS/CFT," JHEP 1006, 061 (2010) [arXiv:1003.0683 [hep-th]].

[33] M. F. Paulos, "New massive gravity, extended," arXiv:1005.1646 [hep-th].

[34] I. Gullu, T. C. Sisman and B. Tekin, "c-functions in the Born-Infeld extended New Massive Gravity," Phys. Rev. D 82, 024032 (2010) [arXiv:1005.3214 [hep-th]].

[35] R. M. Wald, "Black hole entropy is the Noether charge," Phys. Rev. D 48, 3427 (1993) [arXiv:gr-qc/9307038];

V. Iyer and R. M. Wald, "Some properties of Noether charge and a proposal for dynamical black hole entropy," Phys. Rev. D 50, 846 (1994) [arXiv:gr-qc/9403028]; T. Jacobson, G. Kang and R. C. Myers, "On Black Hole Entropy," Phys. Rev. D 49, 6587 (1994) [arXiv:gr-qc/9312023].

[36] T. Jacobson and R. C. Myers, "Black Hole Entropy And Higher Curvature Interactions," Phys. Rev. Lett. 70, 3684 (1993) [arXiv:hep-th/9305016].

[37] R. Emparan, "AdS/CFT duals of topological black holes and the entropy of zero-energy states," JHEP 9906, 036 (1999) [arXiv:hep-th/9906040].

[38] M. Van Raamsdonk, "Comments on quantum gravity and entanglement," arXiv:0907.2939 [hep-th];

M. Van Raamsdonk, "Building up spacetime with quantum entanglement," Gen. Rel. Grav. 42, 2323 (2010) [arXiv:1005.3035 [hep-th]].

[39] T. Nishioka, S. Ryu and T. Takayanagi, "Holographic Entanglement Entropy: An Overview," J. Phys. A 42, 504008 (2009) [arXiv:0905.0932 [hep-th]].

[40] H. Casini, M. Huerta and R. C. Myers, "Towards a derivation of holographic entanglement entropy," arXiv:1102.0440 [hep-th].

[41] See, for example:

P. Calabrese and J. L. Cardy, "Entanglement entropy and quantum field theory," J. Stat. Mech. 0406, P002 (2004) [arXiv:hep-th/0405152];

P. Calabrese and J. L. Cardy, "Entanglement entropy and quantum field theory: A non-technical introduction," Int. J. Quant. Inf. 4, 429 (2006) [arXiv:quant-ph/0505193].

[42] C. G. Callan and F. Wilczek, "On geometric entropy," Phys. Lett. B 333, 55 (1994) [arXiv:hep-th/9401072].

[43] O. Aharony, S. S. Gubser, J. M. Maldacena, H. Ooguri and Y. Oz, "Large N field theories, string theory and gravity," Phys. Rept. 323, 183 (2000) [arXiv:hep-th/9905111]. 
[44] L.-Y. Hung, R. C. Myers and M. Smolkin, unpublished.

[45] D. V. Fursaev and S. N. Solodukhin, "On The Description Of The Riemannian Geometry In The Presence Of Conical Defects," Phys. Rev. D 52, 2133 (1995) [arXiv:hep-th/9501127];

D. V. Fursaev and S. N. Solodukhin, "On one loop renormalization of black hole entropy," Phys. Lett. B 365, 51 (1996) [arXiv:hep-th/9412020].

[46] S. N. Solodukhin, "Entanglement entropy, conformal invariance and extrinsic geometry," Phys. Lett. B 665, 305 (2008) [arXiv:0802.3117 [hep-th]].

[47] M. Banados, C. Teitelboim and J. Zanelli, "Black hole entropy and the dimensional continuation of the Gauss-Bonnet theorem," Phys. Rev. Lett. 72, 957 (1994) [arXiv:gr-qc/9309026];

S. Carlip and C. Teitelboim, "The Off-shell black hole," Class. Quant. Grav. 12, 1699 (1995) [arXiv:gr-qc/9312002];

L. Susskind and J. Uglum, "Black hole entropy in canonical quantum gravity and superstring theory," Phys. Rev. D 50, 2700 (1994) [arXiv:hep-th/9401070].

[48] W. Nelson, "A Comment on black hole entropy in string theory," Phys. Rev. D 50, 7400 (1994) [arXiv:hep-th/9406011].

[49] C. Holzhey, F. Larsen and F. Wilczek, "Geometric and renormalized entropy in conformal field theory," Nucl. Phys. B 424, 443 (1994) [arXiv:hep-th/9403108].

[50] J. L. Cardy and I. Peschel, "Finite Size Dependence Of The Free Energy In Two-Dimensional Critical Systems," Nucl. Phys. B 300, 377 (1988).

[51] C. Fefferman and C. R. Graham, "Conformal Invariants," in Elie Cartan et les Mathématiques d'aujourd'hui (Astérisque, 1985) 95;

C. Fefferman and C. R. Graham, "The Ambient Metric," arXiv:0710.0919 [math.DG].

[52] L. Susskind and E. Witten, "The holographic bound in anti-de Sitter space," arXiv:hep-th/9805114.

[53] L. Susskind, "The World As A Hologram," J. Math. Phys. 36, 6377 (1995) [arXiv:hep-th/9409089];

C. R. Stephens, G. 't Hooft and B. F. Whiting, "Black hole evaporation without information loss," Class. Quant. Grav. 11, 621 (1994) [arXiv:gr-qc/9310006].

[54] R. Bousso, "The holographic principle," Rev. Mod. Phys. 74, 825 (2002) [arXiv:hep-th/0203101].

[55] A. W. Peet and J. Polchinski, "UV/IR relations in AdS dynamics," Phys. Rev. D 59, 065011 (1999) [arXiv:hep-th/9809022]. 
[56] V. K. Dobrev, E. K. Khristova, V. B. Petkova and D. B. Stamenov, "Conformal Covariant Operator Product Expansion Of Two Spin 1/2 Fields," Bulg. J. Phys. 1, 42 (1974);

V. K. Dobrev, G. Mack, V. B. Petkova, S. G. Petrova and I. T. Todorov, "Harmonic Analysis On The N-Dimensional Lorentz Group And Its Application To Conformal Quantum Field Theory," Lecture Notes in Physics 63 (Berlin 1977, 280p);

I. T. Todorov, M. C. Mintchev and V. B. Petkova, "Conformal Invariance In Quantum Field Theory," (Pisa, Italy: Sc. Norm. Sup. 1978, 273p).

[57] A.H. Castro Neto and E.H. Fradkin, "The Thermodynamics of quantum systems and generalizations of Zamolodchikov's C-theorem," Nucl. Phys. B 400, 525 (1993) [arXiv:cond-mat/9301009].

[58] T. Appelquist, A. G. Cohen and M. Schmaltz, "A new constraint on strongly coupled field theories," Phys. Rev. D 60, 045003 (1999) [arXiv:hep-th/9901109];

T. Appelquist, A. G. Cohen, M. Schmaltz and R. Shrock, "New constraints on chiral gauge theories," Phys. Lett. B 459, 235 (1999) [arXiv:hep-th/9904172].

[59] S. Sachdev, "Polylogarithm identities in a conformal field theory in three-dimensions," Phys. Lett. B 309, 285 (1993) [arXiv:hep-th/9305131].

[60] P. Kovtun and A. Ritz, "Black holes and universality classes of critical points," Phys. Rev. Lett. 100, 171606 (2008) [arXiv:0801.2785 [hep-th]];

P. Kovtun and A. Ritz, "Universal conductivity and central charges," Phys. Rev. D 78, 066009 (2008) [arXiv:0806.0110 [hep-th]].

[61] F. Bastianelli, S. Frolov and A. A. Tseytlin, "Conformal anomaly of $(2,0)$ tensor multiplet in six dimensions and AdS/CFT correspondence," JHEP 0002, 013 (2000) [arXiv:hep-th/0001041].

[62] H. Casini and M. Huerta, "A finite entanglement entropy and the c-theorem," Phys. Lett. B 600, 142 (2004) [arXiv:hep-th/0405111];

H. Casini and M. Huerta, "A c-theorem for the entanglement entropy," J. Phys. A 40, 7031 (2007) [arXiv:cond-mat/0610375].

[63] H. Casini and M. Huerta, "Entanglement entropy for the n-sphere," Phys. Lett. B 694, 167 (2010) [arXiv:1007.1813 [hep-th]];

J. S. Dowker, "Hyperspherical entanglement entropy," J. Phys. A 43, 445402 (2010)

[arXiv:1007.3865 [hep-th]].

[64] J. S. Dowker, "Entanglement entropy for even spheres," arXiv:1009.3854 [hep-th].

[65] A. Cappelli and G. D'Appollonio, "On the trace anomaly as a measure of degrees of freedom," Phys. Lett. B 487, 87 (2000) [arXiv:hep-th/0005115]. 
[66] D. N. Kabat, "Black hole entropy and entropy of entanglement," Nucl. Phys. B 453, 281 (1995) [arXiv:hep-th/9503016];

F. Larsen and F. Wilczek, "Renormalization of black hole entropy and of the gravitational coupling constant," Nucl. Phys. B 458, 249 (1996)

[arXiv:hep-th/9506066];

D. V. Fursaev and G. Miele, "Cones, Spins and Heat Kernels," Nucl. Phys. B 484, 697 (1997) [arXiv:hep-th/9605153];

D. Iellici and V. Moretti, "Thermal partition function of photons and gravitons in a Rindler wedge," Phys. Rev. D 54, 7459 (1996) [arXiv:hep-th/9607015];

L. De Nardo, D. V. Fursaev and G. Miele, "Heat-kernel coefficients and spectra of the vector Laplacians on spherical domains with conical singularities," Class. Quant. Grav. 14, 1059 (1997) [arXiv:hep-th/9610011];

V. Moretti and D. Iellici, "Optical approach for the thermal partition function of photons," Phys. Rev. D 55, 3552 (1997) [arXiv:hep-th/9610180].

[67] A. Allais, "Double-trace deformations, holography and the c-conjecture," arXiv:1007.2047 [hep-th].

[68] S. S. Gubser and I. Mitra, "Double-trace operators and one-loop vacuum energy in AdS/CFT," Phys. Rev. D 67, 064018 (2003) [arXiv:hep-th/0210093];

S. S. Gubser and I. R. Klebanov, "A universal result on central charges in the presence of double-trace deformations," Nucl. Phys. B 656, 23 (2003) [arXiv:hep-th/0212138].

[69] J.I. Latorre, C.A. Lutken, E. Rico and G. Vidal, "Fine-grained entanglement loss along renormalization group flows," Phys. Rev. A 71, 034301 (2005) [arXiv:quant-ph/0404120];

G. Vidal, "Entanglement renormalization," Phys. Rev. Lett. 99, 220405 (2007) [arXiv:cond-mat/0512165];

G. Evenbly and G. Vidal, "Entanglement renormalization in free bosonic systems: real-space versus momentum-space renormalization group transforms," New J. Phys. 12, 025007 (2010) [arXiv:0801.2449];

G. Evenbly and G. Vidal, "Entanglement renormalization in noninteracting fermionic systems," Phys. Rev. B 81, 235102 (2010) [arXiv:0710.0692].

[70] M.A. Metlitski, C.A. Fuertes and S. Sachdev, "Entanglement Entropy in the O(N) model," Phys. Rev. B 80, 115122 (2009) [arXiv:0904.4477 [cond-mat]].

[71] B. Hsu, M. Mulligan, E. Fradkin and E.A. Kim, "Universal entanglement entropy in 2D conformal quantum critical points," Phys. Rev. B 79, 115421 (2009) [arXiv:0812.0203].

[72] M. Levin and X. G. Wen, "Detecting Topological Order in a Ground State Wave Function," Phys. Rev. Lett. 96, 110405 (2006) [arXiv:cond-mat/0510613]; 
A. Kitaev and J. Preskill, "Topological entanglement entropy," Phys. Rev. Lett. 96, 110404 (2006) [arXiv:hep-th/0510092].

[73] H. Casini, "Mutual information challenges entropy bounds," Class. Quant. Grav. 24, 1293 (2007) [arXiv:gr-qc/0609126];

H. Casini and M. Huerta, "Remarks on the entanglement entropy for disconnected regions," JHEP 0903, 048 (2009) [arXiv:0812.1773 [hep-th]];

H. Casini and M. Huerta, "Entanglement entropy in free quantum field theory," J. Phys. A 42, 504007 (2009) [arXiv:0905.2562 [hep-th]].

[74] B. Swingle, "Mutual information and the structure of entanglement in quantum field theory," arXiv:1010.4038 [quant-ph].

[75] M. Headrick, "Entanglement Renyi entropies in holographic theories," arXiv:1006.0047 [hep-th].

[76] D. V. Fursaev, "Proof of the holographic formula for entanglement entropy," JHEP 0609, 018 (2006) [arXiv:hep-th/0606184].

[77] L. Y. Hung, R. C. Myers and M. Smolkin, "On Holographic Entanglement Entropy and Higher Curvature Gravity," arXiv:1101.5813 [hep-th];

J. de Boer, M. Kulaxizi and A. Parnachev, "Holographic Entanglement Entropy in Lovelock Gravities," arXiv:1101.5781 [hep-th].

[78] R. C. Myers, S. Sachdev and A. Singh, "Holographic Quantum Critical Transport without Self-Duality," arXiv:1010.0443 [hep-th].

[79] M. F. Paulos, "Holographic phase space: $c$-functions and black holes as renormalization group flows," arXiv:1101.5993 [hep-th].

[80] For example, see:

A. Buchel, R. C. Myers and A. Sinha, "Beyond $\eta / s=1 / 4 \pi$," JHEP 0903, 084 (2009) [arXiv:0812.2521 [hep-th]];

R. C. Myers, M. F. Paulos and A. Sinha, "Holographic Hydrodynamics with a Chemical Potential," JHEP 0906, 006 (2009) [arXiv:0903.2834 [hep-th]].

[81] A. De Felice and S. Tsujikawa, "f(R) theories," Living Rev. Rel. 13, 3 (2010) [arXiv:1002.4928 [gr-qc]].

[82] K. S. Stelle, "Classical Gravity With Higher Derivatives," Gen. Rel. Grav. 9, 353 (1978).

[83] M. Nakasone and I. Oda, "Massive Gravity with Mass Term in Three Dimensions," Phys. Rev. D 79, 104012 (2009) [arXiv:0903.1459 [hep-th]]. 
[84] D. T. Son, "Toward an AdS/cold atoms correspondence: a geometric realization of the Schroedinger symmetry," Phys. Rev. D 78, 046003 (2008) [arXiv:0804.3972 [hep-th]]; K. Balasubramanian and J. McGreevy, "Gravity duals for non-relativistic CFTs," Phys. Rev. Lett. 101, 061601 (2008) [arXiv:0804.4053 [hep-th]].

[85] S. Kachru, X. Liu and M. Mulligan, "Gravity Duals of Lifshitz-like Fixed Points," Phys. Rev. D 78, 106005 (2008) [arXiv:0808.1725 [hep-th]].

[86] A. Adams, A. Maloney, A. Sinha and S. E. Vazquez, "1/N Effects in Non-Relativistic Gauge-Gravity Duality,” JHEP 0903, 097 (2009) [arXiv:0812.0166 [hep-th]].

[87] D. M. Hofman and J. Maldacena, "Conformal collider physics: Energy and charge correlations," JHEP 0805, 012 (2008) [arXiv:0803.1467 [hep-th]].

[88] C. Charmousis, R. Emparan, E. Kiritsis and R. C. Myers, possibly in preparation.

[89] C. Hoyos and P. Koroteev, "On the Null Energy Condition and Causality in Lifshitz Holography," Phys. Rev. D 82, 084002 (2010) [arXiv:1007.1428 [hep-th]]. 